\title{
COMPLETE INTERSECTIONS IN SPHERICAL VARIETIES
}

\author{
KIUMARS KAVEH AND A. G. KHOVANSKII
}

Dedicated to Joseph Bernstein on the occasion of his 70th birthday

\begin{abstract}
Let $G$ be a complex reductive algebraic group. We study complete intersections in a spherical homogeneous space $G / H$ defined by a generic collection of sections from $G$-invariant linear systems. Whenever nonempty, all such complete intersections are smooth varieties. We compute their arithmetic genus as well as some of their $h^{p, 0}$ numbers. The answers are given in terms of the moment polytopes and NewtonOkounkov polytopes associated to $G$-invariant linear systems. We also give a necessary and sufficient condition on a collection of linear systems so that the corresponding generic complete intersection is nonempty. This criterion applies to arbitrary quasi-projective varieties (i.e. not necessarily spherical homogeneous spaces). When the spherical homogeneous space under consideration is a complex torus $\left(\mathbb{C}^{*}\right)^{n}$, our results specialize to well-known results from the Newton polyhedra theory and toric varieties.
\end{abstract}

\section{CONTENTS}

1. Introduction

2. Transversality and complete intersections in general varieties 5

3. Complete intersections in toric varieties 12

4. Virtual polytopes 14

5. Complete intersections in spherical varieties 17

6. Examples 30

References

\section{INTRODUCTION}

The main objective of the present paper is to study complete intersections in a spherical homogeneous space $G / H$ where $G$ is a complex connected reductive algebraic group. We compute the arithmetic genus as well as many of the $h^{p, 0}$ numbers of a generic complete intersection in $G / H$. Our results generalize the similar results from the Newton polyhedra theory and toric varieties obtained in Khovanskii77, Khovanskii78, Khovanskii15.

We first get a convex geometric formula for the Euler characteristic of a $G$-linearized line bundle over a projective spherical variety. This then allows us to represent the arithmetic genus of a complete intersection also in terms of convex geometric data. In many cases of

Date: August 20, 2018.

2010 Mathematics Subject Classification. Primary: 14M27; Secondary: 14M10.

Key words and phrases. Arithmetic and geometric genus, complete intersection, spherical variety, moment polytope, Newton-Okounkov polytope, virtual polytope.

The first author is partially supported by a National Science Foundation Grant (Grant ID: 1200581).

The second author is partially supported by the Canadian Grant No. 156833-12. 
interest our formula is quite computable (Section 6). Our approach is based on the notion of virtual polytope as developed in Khovanskii-Pukhlikov93. Moreover, we use NewtonOkounkov bodies/polytopes associated to linear systems (Okounkov97, Alexeev-Brion04, Kaveh-Khovanskii12b) as well as string polytopes (in particular Gelfand-Zetlin polytopes) associated to irreducible representations of $G$ (Littelmann98, Berenstein-Zelevinsky01] and Kaveh).

Let $G$ be a reductive algebraic group. A variety $X$ with an action of $G$ is called spherical if a Borel subgroup of $G$ has a dense open orbit. Spherical varieties are generalizations of toric varieties (where $G=\left(\mathbb{C}^{*}\right)^{n}$ is a torus) on one hand and the flag varieties $G / P$ on the other hand. Similar to toric varieties, geometry of spherical varieties and their orbit structure can be read off from combinatorial and convex geometric data of fans and convex polytopes.

We begin by recalling results about complete intersections in a torus (see also Section 3). Let $A_{1}, \ldots, A_{k}$ be finite subsets of $\mathbb{Z}^{n}$ where $k \leq n$. For each $i=1, \ldots, k$ let $f_{i}(x)=$ $\sum_{\alpha \in A_{i}} c_{i, \alpha} x^{\alpha}$ be a Laurent polynomial in $\mathbb{C}\left[x_{1}^{ \pm 1}, \ldots, x_{n}^{ \pm 1}\right]$ with generic coefficients $c_{i, \alpha}$. Let

$$
X_{k}=\left\{x \in\left(\mathbb{C}^{*}\right)^{n} \mid f_{1}(x)=\cdots=f_{k}(x)=0\right\}
$$

be the complete intersection in the torus $\left(\mathbb{C}^{*}\right)^{n}$ defined by the $f_{i}$. For each $i=1, \ldots, k$, let $\Delta_{i}$ denote the convex hull of $A_{i}$. It is an integral convex polytope in $\mathbb{R}^{n}$.

In general a generic complete intersection $X_{k}$ in $\left(\mathbb{C}^{*}\right)^{n}$ may be empty. A beautiful result of David Bernstein 1 gives a necessary and sufficient condition for $X_{k}$ to be nonempty, in terms of the dimensions of the polytopes $\Delta_{i}$ and their Minkowski sums. It relies on a theorem of Minkowski which gives a necessary and sufficient condition for the mixed volume of $n$ convex polytopes to be nonzero (see Khovanskii15).

We recall that the arithmetic genus of a smooth complete variety $Z$ of dimension $d$ is by definition $\chi(Z)=\sum_{i=0}^{d}(-1)^{i} h^{i, 0}(Z)$. The geometric genus $p_{g}(Z)$ is the number $h^{d, 0}(Z)$, i.e. the dimension of the space of holomorphic top forms. One knows that $h^{p, 0}$ numbers are birational invariants and hence the $h^{p, 0}$ numbers and the arithmetic and geometric genus can be defined for non-smooth and non-complete varieties as well.

In Khovanskii78 the following formula for the arithmetic genus of $X_{k}$ is proved. It computes the arithmetic genus in terms of the number of integral points in the relative interior of $\Delta_{i}$ and their Minkowski sums:

$$
\chi\left(X_{k}\right)=1-\sum_{i_{1}} N^{\prime}\left(\Delta_{i_{1}}\right)+\sum_{i_{1}<i_{2}} N^{\prime}\left(\Delta_{i_{1}}+\Delta_{i_{2}}\right)-\cdots+(-1)^{k} N^{\prime}\left(\Delta_{1}+\cdots+\Delta_{k}\right),
$$

where for a polytope $\Delta, N^{\prime}(\Delta)$ denotes the number of integral points in the interior of $\Delta$ times $(-1)^{\operatorname{dim}(\Delta)}$. Here the interior is with respect to the topology of the affine span of $\Delta$. Moreover, if all the polytopes $\Delta_{i}$ have full dimension $n$, all the $h^{i, 0}\left(X_{k}\right)$ are 0 except $h^{0,0}\left(X_{k}\right)=1$ and $h^{n-k, 0}\left(X_{k}\right)$ which can be computed from (11). In fact, the condition that the polytopes have full dimension can be substitute with a weaker condition (see Corollary 3.10).

As above let $G$ be a complex connected reductive algebraic group. Let $G / H$ be a spherical homogeneous space of dimension $n$. Let $\mathcal{E}_{1}, \ldots, \mathcal{E}_{k}, k \leq n$, be $G$-linearized globally generated line bundles on $G / H$. For each $i=1, \ldots, k$, let $E_{i} \subset H^{0}\left(G / H, \mathcal{E}_{i}\right)$ be a nonzero $G$-invariant

\footnotetext{
${ }^{1}$ David Bernstein is the younger brother of Joseph Bernstein. He discovered the famous formula for the number of solutions in $\left(\mathbb{C}^{*}\right)^{n}$ of a generic system of $n$ polynomial equations with fixed Newton polytopes Bernstein75. This amazing formula inspired much activity that eventually lead to the creation of Newton polyhedra theory and the theory of Newton-Okounkov bodies.
} 
linear system. That is, $E_{i}$ is a nonzero finite dimensional linear subspace of $H^{0}\left(G / H, \mathcal{E}_{i}\right)$ and stable under the action of $G$. Also for $i=1, \ldots, k$, let $f_{i}$ be a generic section from the linear system $E_{i}$. We are interested in a generic complete intersection:

$$
X_{k}=\left\{x \in G / H \mid f_{1}(x)=\cdots=f_{k}(x)=0\right\} .
$$

Let $\mathcal{E}$ be a $G$-linearized line bundle on $G / H$ and $E \subset H^{0}(G / H, \mathcal{E})$ a nonzero $G$-invariant linear system. Generalizing the notion of Newton polytope of a Laurent polynomial, to $E$ one associates two polytopes: the moment polytope $\Delta(E)$ and the Newton-Okounkov polytope $\tilde{\Delta}(E)$ (see Section [5.4). The moment polytope $\Delta(E)$ is defined as:

$$
\Delta(E)=\overline{\bigcup_{m>0}\left\{\lambda / m \mid V_{\lambda} \text { appears in } \overline{E^{m}}\right\}} .
$$

Here $E^{m} \subset H^{0}\left(G / H, \mathcal{E}^{\otimes m}\right)$ is the linear system spanned by all the products of $m$ elements from $E$ and $\overline{E^{m}}$ is the completion of $E^{m}$ as a linear system. The moment polytope $\Delta(E)$ contains asymptotic information about the highest weights appearing in the complete linear systems $\overline{E^{m}}$ for large $m$. The name moment polytope comes from symplectic geometry since it coincides with the moment polytope in the sense of Hamiltonian group actions (see Remark 5.8). In the context of reductive group actions on varieties, the notion of moment polytope, as defined in (2), goes back to M. Brion ([Brion87).

The Newton-Okounkov polytope $\tilde{\Delta}(E)$ is a polytope fibered over the moment polytope $\Delta(E)$ with string polytopes as fibers (see Section [5.3). It has the property that for each $m>0$, the dimension of the complete linear system $\overline{E^{m}}$ is equal to the number of integral points in the dilated polytope $m \tilde{\Delta}(E)$. From this it follows that the number of intersections of $n$ generic hypersurfaces in the linear system $E$ is equal to $n ! \operatorname{vol}(\tilde{\Delta}(E))$.

The definition of Newton-Okounkov polytope of a spherical variety goes back to A. Okounkov for when $G$ is a classical group (see Okounkov97) and V. Alexeev and M. Brion for a general reductive group (see Alexeev-Brion04). It was generalized to arbitrary $G$-varieties in Kaveh-Khovanskii12b.

The main results of the paper are as follows:

Generalizing the Bernstein's theorem, we give a necessary and sufficient condition for $X_{k}$ to be nonempty. The conditions are in terms of the dimensions of the Newton-Okounkov polytopes of the $E_{i}$ and the products of the $E_{i}$ (see Theorem 5.27). The conditions can also be formulated only in terms of the moment polytopes. In fact, the necessary and sufficient conditions for the nonemptiness of a generic complete intersection can be formulated to apply to an arbitrary quasi-projective variety (see Theorems 2.14 and 2.19).

Moreover, whenever a generic complete intersection $X_{k}$ is not empty, we give a formula for its genus. To $G / H$ there corresponds a sublattice in $\mathbb{Z}^{n}$ and to $E$ there corresponds an integral point $\alpha \in \mathbb{Z}^{n}$. For a polytope $\tilde{\Delta}$ we count the number of points in $\tilde{\Delta}$ lying in the sublattice shifted by $\alpha$. We denote by $N^{\prime}(\tilde{\Delta}, \alpha)$ the number of points in the sublattice shifted by $\alpha$ which lie in the interior of $\tilde{\Delta}$ times $(-1)^{\operatorname{dim}(\tilde{\Delta})}$ (as before the interior is with respect to the topology of the affine span of $\tilde{\Delta})$.

Theorem 1 (Theorem 5.29). The arithmetic genus $\chi\left(X_{k}\right)$ is given by:

$$
\begin{aligned}
\chi\left(X_{k}\right)=1-\sum_{i_{1}} N^{\prime}\left(\tilde{\Delta}_{i_{1}}\left(E_{i_{1}}\right), \alpha_{i_{1}}\right)+\sum_{i_{1}<i_{2}} N^{\prime}\left(\tilde{\Delta}\left(E_{i_{1}} E_{i_{2}}\right), \alpha_{i_{1}}+\alpha_{i_{2}}\right)-\cdots \\
\\
\quad+(-1)^{k} N^{\prime}\left(\tilde{\Delta}\left(E_{1} \cdots E_{k}\right), \alpha_{1}+\cdots+\alpha_{k}\right) .
\end{aligned}
$$

Again the above can also be formulated only in terms of the moment polytopes. 
Finally we give estimates for many of the $h^{i, 0}$ numbers of $X_{k}$ (Theorem 5.32). In particular when all the polytopes $\tilde{\Delta}\left(E_{i}\right)$ have full dimension we have the following:

Theorem 2 (Corollary [5.33). With notation as above, suppose all the polytopes $\tilde{\Delta}\left(E_{i}\right)$, $i=1, \ldots, k$, have full dimension equal to $n=\operatorname{dim}(G / H)$. Then for any $0 \leq p<n-k$ :

$$
h^{p, 0}\left(X_{k}\right)=\left\{\begin{array}{cc}
1, & p=0 \\
0 & p \neq 0
\end{array}\right.
$$

Moreover, $h^{n-k, 0}\left(X_{k}\right)$ can be computed from (3).

In fact, Corollary 5.33 is a slightly stronger version of the above theorem.

In the last section (Section 6) we consider three important classes of spherical varieties (aside from toric varieties): (1) horospherical varieties, (2) group embeddings, and (3) flag varieties. In particular our formula give very computable formulae for the following concrete examples of complete intersections in spherical homogeneous spaces:

- (A horospherical example) Let $V$ be a finite dimensional $G$-module. Let $v_{1}, \ldots, v_{s}$ be highest weight vectors of $V$ with highest weights $\lambda_{1}, \ldots, \lambda_{s}$ respectively. Put $v=v_{1}+\cdots+v_{s}$ and let $X$ be the closure of the $G$-orbit of $v$ in $V$. It is an affine spherical subvariety of $V$. Let $L$ be the linear subspace of $\mathbb{C}[X]$ consisting of linear functions in $V^{*}$ restricted to $X$. Let $\Delta=\operatorname{conv}\left\{\lambda_{i} \mid i=1, \ldots, s\right\}$. Also let $\tilde{\Delta}$ denote the corresponding Newton-Okounkov polytope. Let $f$ be a generic element in $L$ defining a hypersurface $H_{f}=\{x \in X \mid f(x)=0\}$. Then the geometric genus of $H_{f}$ is equal to the number of integral points in the interior of the polytope $\tilde{\Delta}$ (see Section 6.1).

- (A group example) Let $\pi: G \rightarrow \mathrm{GL}(V)$ be a finite dimensional faithful representation of $G$. Let $H$ be a hypersurface in $G$ defined by $f=0$ where $f$ is a generic matrix element of $\pi$. Then the the geometric genus of $H$ is given by the number of points in the interior of the polytope $\tilde{\Delta}_{\pi}$ (see Section 6.2).

- (A flag variety example) Let $G=\operatorname{GL}(n, \mathbb{C})$. Let $\mathcal{L}_{\lambda}$ be the $G$-line bundle on the variety of complete flags associated to a dominant weight $\lambda$. If $H$ is a generic divisor of $\mathcal{L}_{\lambda}$ then the geometric genus of $H$ is equal to the number of integral points in $\mathbb{R}^{n(n-1) / 2}$ lying in the interior of the Gelfand-Zetlin polytope $\Delta_{\mathrm{GZ}}(\lambda)$. In other words, the number of integral points in $\mathbb{R}^{n(n-1) / 2}$ which satisfy the inequalities in (34) where all the inequalities are strict (see Section 6.3).

The second author would like to emphasize that he enjoyed a great amount of support from Joseph Bernstein during his works Khovanskii77, Khovanskii78 and his support played an important role in completion of these papers.

In the late 70's the second author stated and widely advertised the problem of extending the results in Khovanskii77, Khovanskii78, about geometry of complete intersections in a torus $\left(\mathbb{C}^{*}\right)^{n}$ to other reductive groups (see Section 3 ). The first result in this direction was obtained by B. Kazarnovskii (Kazarnovskii87) who computed the number of points in a zero dimensional complete intersection in any reductive group (the answer is expressed as the integral of a certain polynomial over an associated moment polytope). Around the same time, M. Brion generalized Kazarnovskii's result to a zero dimensional complete intersection in any spherical variety (Brion89). Much later the first author showed (Kaveh04) that the straightforward generalization of the formula for the (topological) Euler characteristic of a complete intersection in a torus $\left(\mathbb{C}^{*}\right)^{n}$ to a reductive group fails and such a formula should be more complicated than in the torus case. The corresponding formula was soon 
found by V. Kiritchenko (Kiritchenko06, Kiritchenko07). Her unexpected and beautiful result was at the same time slightly disappointing: the formula turns out to be unavoidably too complicated. This somewhat reduced the hope and suggested that perhaps extensions of formulae for other geometric invariants from the torus case to the reductive case may be too complicated. Nevertheless, in the present paper we give formulae for the arithmetic and geometric genus of complete intersections in a spherical homogeneous space, exactly extending the similar formulae for complete intersections in a torus $\left(\mathbb{C}^{*}\right)^{n}$ in terms of the number of integral points in certain associated polytopes (namely Newton-Okounkov polytopes).

The results of this paper use basic facts about the theory of virtual polytopes and convex chains as developed in Khovanskii-Pukhlikov93. The second author would like to point out that A. Pukhlikov and him arrived at these ideas thinking about the Euler characteritic of $T$-linearized line bundles on toric varieties.

Beside the techniques from Khovanskii77, Khovanskii78, our results strongly rely on the results of Michel Brion on the cohomology of $G$-line bundles on projective spherical varieties (Theorem 5.4), as well as the equivariant resolution of singularities of spherical varieties.

Acknowledgement: We would like to thank Michel Brion for telling us about some references regarding moment polytopes of $G$-varieties.

\section{TRANSVERSALITY AND COMPLETE INTERSECTIONS IN GENERAL VARIETIES}

In this section we discuss some results on transversality and complete intersections in general varieties. We will use them later in Section 5.8 to prove our main results about complete intersection in a spherical homogenous space.

2.1. Stratifications and complete intersections. Let $X$ be a complex quasi-projective algebraic variety. A finite collection $\left\{Y_{i}\right\}$ of its quasi-projective subvarieties is called a stratification of $X$, and each $Y_{i}$ is a stratum, if the following conditions hold: (1) The union of all the strata is $X$. (2) The intersection of any two different strata is empty. (3) Each stratum is a smooth quasi-projective variety.

Our definition of stratification, which suffices for our purposes, requires only mild assumptions on the strata with no condition on how a stratum approaches another stratum on its boundary (for example Whitney A and B conditions in a Whitney stratification). One can prove that any quasi-projective variety admits a stratification.

The following is easy to prove:

Lemma 2.1. Let $X$ be an irreducible quasi-projective $n$-dimensional variety. Then any stratification $\left\{Y_{i}\right\}$ of $X$ has exactly one $n$-dimensional stratum $X_{0}$ and it is dense in $X$.

Consider a local complete intersection $Z$ of codimension $k$ in an $n$-dimensional variety $X$ with a stratification $\left\{Y_{i}\right\}$. The subvariety $Z$ is said to be transverse to a stratum $Y_{j}$ of the stratification if for any point $a \in X \cap Y_{j}$ there is a Zariski open set $U \subset X$, containing $a$, and a system of equations $f_{1}=\cdots=f_{k}=0$ in $U$ defining $Z \cap U$ such that the differentials of the restrictions of $f_{1}, \ldots, f_{k}$ to $Y_{j}$ are independent in the tangent space to $T_{a}\left(Y_{j}\right)$. The subvariety $Z$ is transverse to the stratification $\left\{Y_{i}\right\}$ if it is transverse to all the strata of the stratification.

The following is straightforward:

Theorem 2.2. Let $Z$ be a local complete intersection of codimension $k$ in an $n$-dimensional variety $X$ which is transverse to a stratification $\left\{Y_{i}\right\}$ of $X$. Then: (1) If $Z \cap Y_{j} \neq \emptyset$ the 
variety $Z \cap Y_{j}$ is a smooth local complete intersection of codimension $k$ in $Y_{j}$. (2) The set of all nonempty intersections $\left\{Z \cap Y_{j}\right\}$ form a stratification of $Z$. (3) If $X$ is irreducible then $Z_{0}=Z \cap X_{0}$, where $X_{0}$ is the stratum of dimension $n$, is dense in $Z$. (4) If $X$ is smooth then $Z$ is also smooth.

Let $X$ and $\tilde{X}$ be quasi-projective varieties with stratifications $\left\{Y_{i}\right\},\left\{\tilde{Y}_{j}\right\}$ respectively.

Definition 2.3. A morphism $\pi: \tilde{X} \rightarrow X$ respects the stratifications $\left\{\tilde{Y}_{j}\right\}$ and $\left\{Y_{i}\right\}$ if the following hold: (1) $\pi$ is surjective. (2) Its restriction to each stratum $\tilde{Y}_{j}$ is a surjective map from $\tilde{Y}_{j}$ to some other stratum $Y_{i}$. (3) For every $x \in \tilde{Y}_{j}$ the differential $d \pi_{x}: T_{x} \tilde{Y}_{j} \rightarrow T_{\pi(x)} Y_{i}$ is surjective.

The following is easy to check:

Theorem 2.4. Assume that $\pi: \tilde{X} \rightarrow X$ respects the stratifications $\left\{\tilde{Y}_{j}\right\}$ and $\left\{Y_{i}\right\}$. Let $Z \subset X$ be a local complete intersection of codimension $k$ transverse to the stratification $\left\{Y_{i}\right\}$. Then $\pi^{-1}(Z)=\tilde{Z} \subset \tilde{X}$ is a local complete intersection of codimension $k$ transverse to the stratification $\left\{\tilde{Y}_{j}\right\}$.

Now let $G$ be a complex algebraic group. In this section we consider $G$-varieties, i.e. varieties equipped with an algebraic action of $G$. Any $G$-variety is the union of $G$-orbits. We will always assume that the action of $G$ on the variety has only finitely many orbits. It is clear that then the orbits of $G$ give a stratification of the variety. We refer to this stratification as a $G$-stratification. The following is straightforward.

Lemma 2.5. Let $\pi: \tilde{X} \rightarrow X$ be a $G$-equivariant surjective morphism of $G$-varieties $\tilde{X}$ and $X$. Then $\pi$ respects the $G$-stratifications of $\tilde{X}$ and $X$.

Proof. Consider a point $a \in \tilde{X}$ and its image $b=\pi(a) \in X$. Because $\pi$ is $G$-equivariant it maps the $G$-orbit of the point $a$ onto the $G$-orbit of the point $b$. A tangent vector $\xi_{2}$ at $b$ to the orbit of $b$ is a velocity vector of $b$ under an action of some one-parameter subgroup $G_{1} \subset G_{\text {. }}$. The vector $\xi_{2}$ is the image under $d \pi$ of the velocity vector $\xi_{1}$ at $a$ of the action of $G_{1}$ on $\tilde{X}$. This finishes the proof.

For the sake of completeness we recall some basic theorems about transversality. First we recall the Bertini-Sard theorem. It is an algebraic version of the classical theorem of Sard on critical values of smooth maps on manifolds.

Theorem 2.6 (Bertini-Sard theorem). Let $F: U \rightarrow \mathbb{C}^{k}$ be a morphism from a smooth algebraic variety $U$ to $\mathbb{C}^{k}$ and let $\Sigma \subset \mathbb{C}^{k}$ be the set of critical values of $F$. Then $\Sigma$ is a semi-algebraic subset of $\mathbb{C}^{k}$ of codimension at least one.

We will say that some property holds for a generic point of an irreducible algebraic variety $T$ if there is a proper closed algebraic subset $\Sigma \subset T$ such that the property holds for all the points in $X \backslash \Sigma$ (or equivalently if there is a semialgebraic set of codimension at least one such that the property holds in its complement). In the notation of the Bertini-Sard theorem, the set $F(U) \subset \mathbb{C}^{k}$ is semialgebraic. Therefore according to the theorem a generic point of $\mathbb{C}^{k}$ is not a critical value of $F$, or equivalently, a generic point of $\mathbb{C}^{k}$ is a regular value of $F$.

Lemma 2.7. Suppose $T$ is an irreducible variety. Let $\Sigma \subset T$ be a semi-algebraic subset. Then either $x \in \Sigma$ holds for generic points of $T$, or $x \notin \Sigma$ holds for generic points of $T$.

Proof. If $\operatorname{dim}(\Sigma)=\operatorname{dim}(T)$ then $\operatorname{dim}(T \backslash \Sigma)<\operatorname{dim}(T)$. If $\operatorname{dim}(\Sigma)<\operatorname{dim}(T)$ then $\operatorname{dim}(T \backslash$ $\Sigma)=\operatorname{dim}(T)$. This proves the lemma. 
Let $\mathcal{L}_{1}, \ldots, \mathcal{L}_{k}$ be line bundles on $X$, and for $i=1, \ldots, k$ let $E_{k} \subset H^{0}\left(X, \mathcal{L}_{i}\right)$ be a finite dimensional linear subspace of sections. Let $\mathbf{E}$ denote the $k$-fold product $E_{1} \times \cdots \times E_{k}$. The following is an immediate corollary of Lemma 2.7

Lemma 2.8. Let $Z_{\mathrm{g}} \subset X$ be the subvariety defined by $g_{1}=\cdots=g_{k}=0$ for some $\mathbf{g}=\left(g_{1}, \ldots, g_{k}\right) \in \mathbf{E}$. Then either $Z_{\mathbf{g}}$ is empty for generic $\mathbf{g} \in \mathbf{E}$, or $Z_{\mathbf{g}}$ is nonempty for generic $\mathbf{g} \in \mathbf{E}$.

With notation as in Lemma 2.8 we have the following version of the Thom transversality theorem. It is a corollary of the Bertini-Sard theorem. We skip the details.

Theorem 2.9 (A version of Thom's transversality theorem). Let $X$ be a quasi-projective variety equipped with a stratification $\left\{Y_{i}\right\}$. Assume that the following hold: (1) The linear systems $E_{1}, \ldots, E_{k}$ are base point free, and (2) $Z_{\mathbf{g}} \neq \emptyset$ for generic $\mathbf{g} \in \mathbf{E}=E_{1} \times \cdots \times E_{k}$. Then, for generic $\mathbf{g} \in \mathbf{E}$, the subvariety $Z_{\mathbf{g}}$ is a local complete intersection of codimension $k$ which is transverse to $\left\{Y_{i}\right\}$.

2.2. When is a generic complete intersection nonempty? Suppose we are given $k$ linear systems on a variety. In this section we give a necessary and sufficient condition for a generic complete intersections from these linear systems to be nonempty. We would need the notion of the Kodaira map of a linear system which we briefly explain below:

As above let $X$ be an $n$-dimensional quasi-projective variety. Let $E$ be a linear system on $X$, that is, a finite dimensional linear subspace of global sections of a line bundle $\mathcal{L}$ on $X$. Assume that $E$ is base point free. One can then define a morphism $\Phi_{E}: X \rightarrow \mathbb{P}\left(E^{*}\right)$ called the Kodaira map of $E$. It is defined as follows: $\Phi_{E}(x)$ is the point in the projective space $\mathbb{P}\left(E^{*}\right)$ represented by the hyperplane $H_{x}$ in $E$ consisting of all the sections which vanish at $x$. We denote the closure of the image of $\Phi_{E}$ by $Y_{E}$. It is a projective subvariety of $\mathbb{P}\left(E^{*}\right)$. The following is easy to prove from the definition of the Kodaira map:

Lemma 2.10. For $a, b \in X$ we have $\Phi_{E}(a)=\Phi_{E}(b)$, if and only if the sets $\{g \in E \mid g(a)=$ $0\}$ and $\{g \in E \mid g(b)=0\}$ coincide.

The notion of a Kodaira map is very useful in the theory of Newton-Okounkov bodies. One usually assumes that the linear system under consideration is large enough so that the Kodaira map $\Phi_{E}$ is an isomorphism (or at least a birational isomorphism) between $X$ and $Y_{E}$. In this section we relax this and would work with general base point free linear systems $E$ such that $Y_{E}$ can have smaller dimension than that of $X$.

Let $\mathcal{L}_{1}, \ldots, \mathcal{L}_{k}$ be a collection of globally generated line bundles on $X$. For $i=1, \ldots, k$ let $E_{i} \subset H^{0}\left(X, \mathcal{L}_{i}\right)$ be a finite dimensional subspaces of global sections of $\mathcal{L}_{i}$ without base point. We will use the following notation: $I$ denotes the set of indices $\{1, \ldots, k\}$ and $J=\left\{i_{1}, \ldots, i_{j}\right\}$ is a nonempty subset of $I$. We write $\mathcal{L}_{J}$ for the line bundle $\mathcal{L}_{i_{1}} \otimes \cdots \otimes \mathcal{L}_{i_{j}}$ and $E_{J}$ is the subspace of $H^{0}\left(X, \mathcal{L}_{J}\right)$ spanned by all the tensor products $g_{i_{1}} \otimes \cdots \otimes g_{i_{j}}$, where $g_{i_{\ell}}$ is a section of $E_{i_{\ell}}$ for $\ell=1, \ldots, j$. We will denote the Kodaira map of the linear system $E_{J}$ simply by $\Phi_{J}: X \rightarrow \mathbb{P}\left(E_{J}^{*}\right)$. We have the following extension of Lemma 2.10

Lemma 2.11. For $a, b \in X$ we have $\Phi_{I}(a)=\Phi_{I}(b)$ if and only if for every $i \in I$ the sets $\left\{g_{i} \in E_{i} \mid g_{i}(a)=0\right\}$ and $\left\{g_{i} \in E_{i} \mid g_{i}(b)=0\right\}$ coincide.

Proof. Let us prove that if $\Phi_{I}(a)=\Phi_{I}(b)$ and $g_{i}(a)=0$ for some $g_{i} \in E_{i}$ then $g_{i}(b)=0$. For every $j \neq i$ fix a section $f_{j} \in E_{j}$ such that $f_{j}(a) \neq 0$ and $f_{j}(b) \neq 0$. For any $g_{i} \in E_{i}$ consider the section $\phi=f_{1} \otimes \cdots \otimes f_{i-1} \otimes g_{i} \otimes f_{i+1} \otimes \cdots \otimes f_{k} \in E_{I}$. By Lemma 2.10 the conditions $\phi(a)=0$ and $\phi(b)=0$ are equivalent. So the equations $g_{i}(a)=0$ and $g_{i}(b)=0$ on a section 
$g_{i} \in E_{i}$ are equivalent. Conversely, assume that for every $i$ the equations $g_{i}(a)=0$ and $g_{i}(b)=0$ for $g_{i} \in E_{i}$ are equivalent. Represent each linear space $E_{i}$ in the form $E_{i}^{0} \oplus E_{i}^{1}$ where every section from $E_{i}^{0}$ vanishes at the points $a$ and $b$ and the one-dimensional subspace $E_{i}^{1}$ is spanned by a section $s_{i}$ not vanishing at $a$ and $b$. The linear space $E_{I}$ is a sum of $2^{k}$ subspaces $E_{I}^{n_{1}, \ldots, n_{k}}$ where the sum is taken over all $2^{k} k$-tuples $\left(n_{1}, \ldots, n_{k}\right)$ of indexes $n_{i}=0,1$, and each linear space $E_{I}^{n_{1}, \ldots, n_{k}}$ is spanned by the tensor products $f_{1} \otimes \cdots \otimes f_{k}$ where $f_{i} \in E_{i}^{0}$ if $n_{i}=0$ and $f_{i}=s_{i}$ if $n_{i}=1$. The sections belonging to each summand but not to the one-dimensional subspace $E_{I}^{1, \ldots, 1}$ spanned by the section $s_{1} \otimes \cdots \otimes s_{k}$ vanish at $a$ and $b$. So the conditions $\phi(a)=0$ and $\phi(b)=0$ for $\phi \in E_{I}$ are equivalent. By Lemma 2.10 we have $\Phi_{I}(a)=\Phi_{I}(b)$ which finishes the proof.

Definition 2.12. With notation as above, we define the defect $d(J)$ of a subset $J \subset I=$ $\{1, \ldots, k\}$ to be the number:

$$
d(J)=\tau_{J}-|J|,
$$

where $\tau_{J}$ is the dimension of $Y_{J}$, the closure of the image of $X$ under the Kodaira map $\Phi_{J}$ and $|J|$ is the number of elements in $J$.

Definition 2.13. With notation as above, we say that the linear systems $E_{1}, \ldots, E_{k}$ are independent if any subset $J \subset I=\{1, \ldots, k\}$ has nonnegative defect.

Theorem 2.14 (Necessary condition for a generic complete intersection to be nonempty). Let $E_{1}, \ldots, E_{k}$ be base point free linear systems. Suppose $Z_{\mathbf{g}} \subset X$ is nonempty for a generic choice of $\mathbf{g} \in \mathbf{E}=E_{1} \times \cdots \times E_{k}$. Then $E_{1}, \ldots, E_{k}$ are independent (in the sense of Definition 2.13).

Proof. Assume that the set $J=\left\{i_{1}, \ldots, i_{j}\right\}$ has a negative defect $d(J)$ with respect to the collection of linear systems $E_{1}, \ldots, E_{k}$. Let us show that for a generic choice of $\mathbf{g}_{J}=$ $\left(g_{i_{1}}, \ldots, g_{i_{k}}\right) \in E_{i_{1}} \times \cdots \times E_{i_{j}}$, the subvariety $Z_{\mathbf{g}_{J}} \subset X$ defined by the equations $g_{i_{1}}=$ $\cdots=g_{i_{k}}=0$ is empty. Suppose this is not the case. Fix a stratification of $X$ with the largest stratum $X_{0}$. Then by Theorem 2.9 the variety $Z_{\mathbf{g}_{J}}$ is a local complete intersection of dimension $n-|J|$ and the intersection $Z_{\mathbf{g}_{J}}^{0}=Z_{\mathbf{g}_{J}} \cap X_{0}$ is nonempty and smooth . Let $a$ be a point in $Z_{\mathbf{g}_{J}}^{0}$. By Lemma 2.11 the smooth variety $Z_{\mathbf{g}_{J}}^{0}$ should contain the set of all points $x \in X_{0}$ such that $\Phi_{J}(a)=\Phi_{J}(x)$. The dimension of the set $K_{a}=\Phi_{J}^{-1}\left(\Phi_{J}(a)\right) \cap X_{0}$ is greater than or equal to $n-\tau_{J}$. Indeed, $\Phi_{J}$ restricted to $X_{0}$ is a surjective morphism from the smooth variety $X_{0}$ of dimension $n$ to the variety $\Phi_{J}\left(X_{0}\right)$ of dimension $\tau_{J}$. But $d(J)=\tau_{J}-|J|<0$ so $n-\tau_{J}>n-|J|$ which is impossible because $K_{a} \subset Z_{\mathbf{g}_{J}}^{0}$. The contradiction proves that $Z_{\mathbf{g}_{J}}$ is empty. This shows that $Z_{\mathbf{g}}$ is empty as well.

The rest of this section is devoted to proving the converse of Theorem 2.14 (Theorem 2.19).

First we introduce a foliation on $X$ using the Kodaira map of a linear system. Let $E$ be a base point free finite dimensional linear subspace of global sections of a line bundle $\mathcal{L}$ on $X$. Let $\Phi_{E}: X \rightarrow \mathbb{P}\left(E^{*}\right)$ be the corresponding Kodaira map. Below we use the following notation: $S$ is the singular locus of the variety $X$. The number $\tau_{E}$ is the dimension of $Y_{E}$, the closure of the image of the Kodaira map $\Phi_{E}$. The set $S_{E}$ is the singular locus of $Y_{E}$ and $U$ is the Zariski open set $X \backslash\left(S \cup \Phi_{E}^{-1}\left(S_{E}\right)\right)$ in $X$. Finally, $\Sigma_{c} \subset U$ is the set of critical points of $\Phi_{E}$ restricted to $U \subset X$.

Definition 2.15. Let $a \in U \backslash \Sigma_{c}$. Let $\tilde{F}(a)$ be the subspace of the tangent space $T_{a} U$ defined by the linear equations $d g_{a}=0$ for all $g \in E$. The collection of subspaces $\tilde{F}(a)$ defines an $\left(n-\tau_{E}\right)$-dimensional distribution $\tilde{F}$ on the Zariski open set $U \backslash \Sigma_{c}$ in $X$. 
The next lemma is a corollary of the Implicit Function Theorem.

Lemma 2.16. The foliation $\tilde{F}$ in $U \backslash \Sigma_{c}$ is completely integrable. Its leaves are connected components of the preimages under the Kodaira map $\Phi_{E}: U \backslash \Sigma_{c} \rightarrow Y_{E}$ of the points in $\Phi_{E}\left(U \backslash \Sigma_{c}\right)$.

Take a point $a \in U \backslash \Sigma_{c}$ and a section $g \in E$ such that $g(a)=0$ and $d g(a) \neq 0$. Let $H$ be the hyperplane in the tangent space $T_{a} U$ defined by $d g=0$. The following is straightforward:

Lemma 2.17. 1) The hyperplane $H$ contains the $\left(n-\tau_{E}\right)$-plane $\tilde{F}(a)$. 2) For any hyperplane $H \subset T_{a} U$ which contains the plane $\tilde{F}(a)$ there is a section $g \in E$ such that $g(a)=0$ and $H$ is defined by the equation $d g_{a}=0$.

We will need a linear algebra statement about transversality of a collection of hyperplanes. Let $F_{1}, \ldots, F_{k} \subset T$ be subspaces in a vector space $T$. For any nonempty set $J=\left\{i_{1}, \ldots, i_{j}\right\} \subset\{1, \ldots, k\}$ let $F_{J}$ denote the the subspace $F_{i_{1}} \cap \cdots \cap F_{i_{j}}$. The following theorem gives a necessary and sufficient condition for the existence of hyperplanes $H_{1}, \ldots, H_{k} \subset T$ such that: (1) $F_{i} \subset H_{i}$ for $1 \leq i \leq k$, and (2) the hyperplanes $H_{1}, \ldots, H_{k}$ are mutually transverse.

Theorem 2.18. The hyperplanes $H_{1}, \ldots, H_{k}$ satisfying the above conditions exist if and only if for any subset $J$ the codimension of $F_{J}$ in $T$ is greater than or equal to $|J|$.

Proof. First suppose the subspaces $H_{1}, \ldots, H_{k}$ satisfying the above conditions exist. For any nonempty subset $J=\left\{i_{1}, \ldots, i_{j}\right\}$ let $H_{J}$ denote the subspace $H_{i_{1}} \cap \cdots \cap H_{i_{j}}$. Then $F_{J} \subset H_{J}$ and the codimension of $H_{J}$ in $T$ is $|J|$. So the codimension of $F_{J}$ is greater than or equal to $|J|$. We prove the converse statement by induction on $k$. Suppose that the statement holds for any collection of $(k-1)$ subspaces. Choose $H_{k}$ to be a hyperplane such that $H_{k}$ contains $F_{k}$ but it does not contain any subspace $F_{J}$ not inside $F_{k}$ (so $F_{J} \cap H_{k}$ has codimension 1 in $F_{J}$ ). Now we apply the induction hypothesis to the $k-1$ hyperplanes $F_{i}^{\prime}=F_{i} \cap H_{k}, i=1, \ldots, k-1$, in the vector space $H_{k}$. Let us verify that these satisfy the conditions in the theorem. For any subset $J \subset\{1, \ldots, k\}$ let $J^{*}=J \backslash\{k\}$. If $F_{J^{*}} \subset F_{k}$ then $F_{J^{*}}=F_{J^{*}} \cap F_{k}=F_{J}$ where $J=J^{*} \cap\{k\}$. The codimension of $F_{J^{*}} \cap H_{k}$ in $H_{k}$ is equal to the codimension of $F_{J}$ in $T$ minus 1 . By the assumption, $\operatorname{codim}\left(F_{J}\right)-1$ is greater than or equal to $\left(\left|J^{*}\right|+1\right)-1=\left|J^{*}\right|$. On the other hand, if $F_{J^{*}}$ is not contained in $F_{k}$ then its codimension in $T$ is equal to the codimension of $F_{J^{*}} \cap H_{k}$ in $H_{k}$. Again by the assumption in the theorem this is greater than or equal to $\left|J^{*}\right|$. Finally by the induction hypothesis there are mutually transverse hyperplanes $H_{1}^{\prime}, \ldots, H_{k-1}^{\prime}$ in $H_{k}$, such that $F_{i} \cap H_{k} \subset H_{i}^{\prime}$ for $i<k$. Enlarge each $H_{i}^{\prime}$ to a hyperplane $H_{i}$ in $T$ such that $H_{i}$ contains $F_{i}$. The collection of hyperplanes $H_{1}, \ldots, H_{k}$ has the required properties.

We can now prove the converse of Theorem 2.14,

Theorem 2.19 (Sufficient condition for a generic complete intersection to be nonempty). Let $E_{1}, \ldots, E_{k}$ be a collection of base point free linear systems. Suppose that $E_{1}, \ldots, E_{k}$ are independent in the sense of Definition 2.13. Then a generic complete intersection $Z_{\mathbf{g}} \subset X$, where $\mathbf{g} \in \mathbf{E}=E_{1} \times \cdots E_{k}$, is nonempty.

Proof. Let $J=\left\{i_{1}, \ldots, i_{j}\right\}$ be a nonempty subset of $I=\{1, \ldots, k\}$. Consider the Kodaira map $\Phi_{J}$ associated with the space $E_{J}$. Using Lemma 2.16] one can find a smooth Zariski open subst $U \subset X$ and integrable foliations $\tilde{F}_{J}$ in $U$ of codimensions $\tau_{J}$ whose leaves are connected components of the manifolds $\Phi_{J}^{-1}(b) \cap U$ where $b \in \Phi_{J}(U)$. Take a point $a \in U$ and let $T=T_{a} U$ be the tangent space to $U$ at $a$. Let $F_{1}, \ldots, F_{k}$ be the subspaces in $T$ 
tangent to the leaves of these foliations. For any nonempty $J=\left\{i_{1}, \ldots, i_{j}\right\}$ the intersection $F_{J}=F_{i_{1}} \cap \cdots \cap F_{i_{j}} \subset T$ coincides with the subspace tangent to the leaf of the foliation $\tilde{F}_{J}$ passing through $a$ (Lemma 2.11). The codimension $\tau_{J}$ of $\tilde{F}_{J}$ in $T$ is $d(J)+|J| \geq|J|$. Thus applying Theorem 2.18 we can find mutually transverse hyperplanes $H_{1} \ldots, H_{k} \subset T$ such that $\tilde{F}_{i} \subset H_{i}$ for $i=1, \ldots, k$. By Lemma 2.17 there are sections $g_{i} \in E_{i}, i=1, \ldots, k$ such that $g_{i}(a)=0$ and the tangent hyperplane to $\left\{g_{i}=0\right\}$ at $a$ is $\tilde{F}_{i}$. Hence the hypersurfaces $g_{i}=0$ at a neighborhood of the point $a$ are smooth and mutually transverse. By the Implicit Function Theorem we then know that for a $k$-tuple of sections $\tilde{\mathbf{g}}=\left(\tilde{g}_{1}, \ldots, \tilde{g}_{k}\right)$ close enough to $g$ the variety $Z_{\tilde{g}}$ is nonempty. Thus it is not true that for a generic $\mathbf{g}$ the variety $Z_{\mathbf{g}}$ is empty. This finishes the proof of the theorem.

2.3. The $h^{p, 0}$ numbers of a complete intersection. The material in this section are taken from Khovanskii15. Let $\mathcal{L}$ be a line bundle on a smooth projective variety $X$ and let $D$ be a divisor of $\mathcal{L}$. We denote by $\mathcal{O}(X, \mathcal{L})$ or $\mathcal{O}(X, D)$ the germ of regular sections of $\mathcal{L}$. We denote the $i$-th sheaf cohomology group of $X$ with coefficients in the sheaf $\mathcal{O}(X, \mathcal{L})$ by $H^{i}(X, \mathcal{L})$ or $H^{i}(X, D)$. We will write $H^{i}(X)$ for the $i$-th cohomology group of the zero divisor, that is, $H^{i}(X)$ is the $i$-th cohomology of $X$ with coefficients in $\mathcal{O}_{X}$, the sheaf of germs of regular functions. For an integer $p \geq 0$, we have $\operatorname{dim}\left(H^{p}(X)\right)=h^{p, 0}(X)$, the dimension of the space of holomorphic $p$-forms on $X$. In particular, $h^{0,0}(X)$ is the number of irreducible components of $X$ and $h^{p, 0}(X)=0$ for $p>\operatorname{dim}(X)$.

Fix a collection $\mathcal{L}_{1}, \ldots, \mathcal{L}_{k}$ of globally generated line bundles on $X$. Recall that a line bundle is globally generated if for any $x \in X$ there is a global section that does not vanish at $x$. For each $i=1, \ldots, k$ let $f_{i} \in H^{0}\left(X, \mathcal{L}_{i}\right)$ be such that the divisor $D_{i}$ defined by $f_{i}=0$ is a smooth hypersurface. Moreover, assume that the divisors $D_{1}, \ldots, D_{k}$ intersect transversely. We will be interested in the local complete intersection $X_{k}=D_{1} \cap \cdots \cap D_{k}$. From transversality it follows that this intersection is a smooth subvaritey of $X$.

Given the dimensions of the cohomology groups:

$$
H^{i}\left(X, \mathcal{L}_{1}^{\otimes m_{1}} \otimes \cdots \otimes \mathcal{L}_{k}^{\otimes m_{k}}\right), \quad m_{1}, \ldots, m_{k} \in\{0,-1\},
$$

of the ambient variety $X$, one can obtain much information about the $h^{p, 0}$ numbers of the complete intersection $X_{k}$. In particular one can compute the arithmetic genus of $X_{k}$. Below we recall how this can be done (Khovanskii78).

For $m=1, \ldots, k$ let $X_{m}=D_{1} \cap \cdots \cap D_{m}$. We then have a sequence of smooth subvarieties $X_{k} \subset \cdots \subset X_{0}=X$ where each variety is a hypersurface in the next one. For a line bundle $\mathcal{L}$ consider the exact sequence of sheaves:

$$
0 \rightarrow \mathcal{O}\left(X_{m-1}, \mathcal{L} \otimes \mathcal{L}_{m}^{-1}\right) \stackrel{i}{\rightarrow} \mathcal{O}\left(X_{m-1}, \mathcal{L}\right) \stackrel{j}{\rightarrow} \hat{\mathcal{O}}\left(X_{m-1}, \mathcal{L}\right) \rightarrow 0 .
$$

Here $\mathcal{O}\left(X_{m-1}, \mathcal{L} \otimes \mathcal{L}_{m}^{-1}\right)$ is the sheaf on $X_{m-1}$ of regular sections of the line bundle $\mathcal{L} \otimes \mathcal{L}_{m}^{-1}$ on $X_{m-1}$. The sheafs $\mathcal{O}\left(X_{m-1}, \mathcal{L}\right)$ and $\mathcal{O}\left(X_{m}, \mathcal{L}\right)$ have analogues definitions. The sheaf $\hat{\mathcal{O}}\left(X_{m-1}, \mathcal{L}\right)$ is the trivial extension of the sheaf $\mathcal{O}\left(X_{m}, \mathcal{L}\right)$ on $X_{m}$ to a sheaf on $X_{m-1}$. The homomorphism $i$ maps a section $g$ of $\mathcal{L} \otimes \mathcal{L}_{m}^{-1}$ to a section $g \otimes f_{m}$ where $f_{m}$ is a global section of $\mathcal{L}_{m}$ defining the divisor $D_{m}$, and the homomorphism $j$ at $a \in X_{m}$ maps a section of $\mathcal{L}$ on $X_{m-1}$ to its restriction to $X_{m}$, and at a point $a \in X_{m-1} \backslash X_{m}$ the homomorphism $j$ is trivial. The long exact sequence of the cohomology groups corresponding to the above exact sequence of sheaves is as follows:

$$
0 \rightarrow H^{0}\left(X_{m-1}, \mathcal{L} \otimes \mathcal{L}_{m}^{-1}\right) \rightarrow H^{0}\left(X_{m-1}, \mathcal{L}\right) \rightarrow H^{0}\left(X_{m}, \mathcal{L}\right) \rightarrow \cdots,
$$

(the cohomology of the sheaves $\mathcal{O}\left(X_{m}, \mathcal{L}\right)$ and $\hat{\mathcal{O}}\left(X_{m-1}, \mathcal{L}\right)$ are canonically isomorphic). 
For a complete variety $X$ with a sheaf $\mathcal{F}$ we denote the Euler characteristic of $X$ with coefficients in $\mathcal{F}$ by $\chi(X, \mathcal{F})$ :

$$
\chi(X, \mathcal{F})=\sum_{i=0}^{n}(-1)^{i} \operatorname{dim}\left(H^{i}(X, \mathcal{F})\right) .
$$

In particular, we write $\chi(X, \mathcal{L})$ for the Euler characterisitic of $X$ with coefficients in the sheaf $\mathcal{O}(X, \mathcal{L})$ of sections of a line bundle $\mathcal{L}$.

The Euler characteristic is additive, i.e. if $\mathcal{G}$ is a sub-sheaf of sheaf $\mathcal{F}$ on $X$ then:

$$
\chi(X, \mathcal{F})=\chi(X, \mathcal{G})+\chi(X, \mathcal{F} / \mathcal{G}),
$$

where $\mathcal{F} / \mathcal{G}$ is the quotient sheaf. The exact sequence (4) then allows us to find the Euler characteristic $\chi\left(X_{k}, \mathcal{L}\right)$. We give the answer for the trivial bundle, that is, $\chi\left(X_{k}\right)$ :

Theorem 2.20. The arithmetic genus $\chi\left(X_{k}\right)$ of the smooth variety $X_{k}$ is equal to:

$$
\chi(X)-\sum_{i_{1}} \chi\left(X, \mathcal{L}_{i}^{-1}\right)+\sum_{i_{1}<i_{2}} \chi\left(X, \mathcal{L}_{i_{1}}^{-1} \otimes \mathcal{L}_{i_{2}}^{-1}\right)-\cdots+(-1)^{k} \chi\left(X, \bigotimes_{1 \leq i \leq k} \mathcal{L}_{i}^{-1}\right) .
$$

For a nonempty set $J \subset\{1, \ldots, k\}$ let $\mathcal{L}_{J}^{-1}=\bigotimes_{i \in J} \mathcal{L}_{i}^{-1}$.

Theorem 2.21. We have the following upper bound for the $h^{i, 0}$ numbers of the complete intersection $X_{k}$ :

$$
h^{i, 0}\left(X_{k}\right) \leq h^{i, 0}(X)+\sum_{J \neq \emptyset} \operatorname{dim}\left(H^{i+|J|}\left(X, \mathcal{L}_{J}^{-1}\right)\right) .
$$

Proof. We can rewrite (5) as $h^{i}\left(X_{k}\right) \leq \sum_{J} \operatorname{dim}\left(H^{i+|J|}\left(X, \mathcal{L}_{J}^{-1}\right)\right)$. Let $\mathcal{L}$ be any line bundle on $X$. We will prove the following more general inequality which coincides with (5) when $\mathcal{L}$ is the trivial line bundle:

$$
\operatorname{dim}\left(H^{i}\left(X_{k}, \mathcal{L}\right)\right) \leq \sum_{J} \operatorname{dim}\left(H^{i+|J|}\left(X, \mathcal{L} \otimes \mathcal{L}_{J}^{-1}\right)\right) .
$$

We prove (6) by induction on $k$. Let $j \geq 0$ and $1 \leq m \leq k$. From the piece:

$$
\rightarrow H^{j}\left(X_{m-1}, \mathcal{L}\right) \rightarrow H^{j}\left(X_{m}, \mathcal{L}\right) \rightarrow H^{j+1}\left(X_{m-1}, \mathcal{L} \otimes \mathcal{L}_{m}^{-1}\right) \rightarrow \ldots
$$

of the exact sequence (4) we obtain that:

$$
\operatorname{dim}\left(H^{j}\left(X_{m}, \mathcal{L}\right)\right) \leq \operatorname{dim}\left(H^{j}\left(X_{m-1}, \mathcal{L}\right)\right)+\operatorname{dim}\left(H^{j+1}\left(X_{m-1}, \mathcal{L} \otimes \mathcal{L}_{m}^{-1}\right)\right) .
$$

For $k=1$, the inequality (6) coincides with (7) for $j=i$ and $m=1$. Assume that (6) is proved for $k-1$. For $J \subset\{1, \ldots, k\}$ let $J^{*}=J \cap\{1, \ldots, k-1\}$. Then either $J=J^{*}$ or $J=J^{*} \cup\{k\}$. In the first case we have:

$$
|J|=\left|J^{*}\right| \text { and } \mathcal{L} \otimes \mathcal{L}_{J}^{-1}=\mathcal{L} \otimes \mathcal{L}_{J^{*}}^{-1} .
$$

In the second case we have:

$$
|J|=\left|J^{*}\right|+1 \text { and } \mathcal{L} \otimes \mathcal{L}_{J}^{-1}=\mathcal{L} \otimes \mathcal{L}_{J^{*}}^{-1} \otimes \mathcal{L}_{k}^{-1} .
$$

By induction hypothesis we can assume that for any line bundle $\mathcal{L}$ and $i \geq 0$ the following inequality holds:

$$
\operatorname{dim}\left(H^{i}\left(X_{k-1}, \mathcal{L}\right)\right) \leq \sum_{J^{*}} \operatorname{dim}\left(H^{i+\left|J^{*}\right|}\left(X, \mathcal{L} \otimes \mathcal{L}_{J^{*}}^{-1}\right)\right),
$$


where the summation is over all the subsets $J^{*} \subset\{1, \ldots, k-1\}$. Also by induction hypothesis we know that for the line bundle $\mathcal{L} \otimes \mathcal{L}_{k}^{-1}$ and any $i+1$ the inequality:

$$
\operatorname{dim}\left(H^{i+1}\left(X_{k-1}, \mathcal{L} \otimes \mathcal{L}_{k}^{-1}\right)\right) \leq \sum_{J^{*}} \operatorname{dim}\left(H^{i+1+\left|J^{*}\right|}\left(X, \mathcal{L} \otimes \mathcal{L}_{k}^{-1} \otimes \mathcal{L}_{J^{*}}^{-1}\right)\right)
$$

holds. Here also the summation is taken over all subsets $J^{*} \subset\{1, \ldots, k-1\}$. Now instead of the numbers $\operatorname{dim}\left(H^{i}\left(X_{k-1}, \mathcal{L}\right)\right)$ and $\operatorname{dim}\left(H^{i+1}\left(X_{k-1}, \mathcal{L} \otimes \mathcal{L}_{k}^{-1}\right)\right)$ plug the righthand sides of (10) and (11) into (7). Using (8) and (9) we obtain the required inequality (6). The theorem is proved.

We have the following direct corollary of Theorem 2.21,

Corollary 2.22. Assume that for some integer $i$ the cohomology groups $H^{i+|J|}\left(X, \mathcal{L}_{J}^{-1}\right)$ vanish for any nonempty $J \subset\{1, \ldots, k\}$. Then $h^{i, 0}\left(X_{k}\right) \leq h^{i, 0}(X)$.

\section{Complete intersections in toric VARIETIES}

The material in this section are taken from Khovanskii78 and Khovanskii15. We explain the results on genus of complete intersections in torus $\left(\mathbb{C}^{*}\right)^{n}$ from Khovanskii78 concerning the case when the Newton polytopes involved are full dimensional, as well as their extensions in Khovanskii15] to the case when the polytopes are not necessarily full dimensional.

Let $\Delta_{1}, \ldots, \Delta_{k}$ be integral polytopes in $\mathbb{R}^{n}$ (i.e. with vertices in $\mathbb{Z}^{n}$ ). Let $f_{1}, \ldots, f_{k}$ be a generic $k$-tuple of Laurent polynomials with Newton polytopes $\Delta_{1}, \ldots, \Delta_{k}$ respectively. Let $Z$ be the subvariety of $\left(\mathbb{C}^{*}\right)^{n}$ defined by $f_{1}=\cdots=f_{k}=0$.

Let $X$ be a smooth projective toric variety which is sufficiently complete with respect to the polytopes $\Delta_{1}, \ldots, \Delta_{k}$ in the sense of Khovanskii77. We let $D_{\infty}$ denote the divisor at infinity of a generic Laurent polynomial with Newton polytope $\Delta=\Delta_{1}+\cdots+\Delta_{k}$ on $X$. It is a divisor supported on the complement of the open orbit $\left(\mathbb{C}^{*}\right)^{n}$ and hence is torus invariant. Let $\mathcal{L}_{\infty}$ be the line bundle corresponding to $D_{\infty}$.

Since the divisor $D_{\infty}$ is torus invariant one can use theory of toric varieties to compute the sheaf cohomology groups of $D_{\infty}$ in terms of the polytope $\Delta$ ([Khovanskii77, Section 4]). We recall the answer below. We need a bit of notation: for a polytope $\Delta$ we denote the number of integral points in $\Delta$ by $N(\Delta)$. Also $N^{\circ}(\Delta)$ denotes the number of integral points in the interior of the polytope $\Delta$. Here the interior is with respect to the topology of the affine span of $\Delta$. We also write $N^{\prime}(\Delta)$ for $(-1)^{\operatorname{dim}(\Delta)} N^{\circ}(\Delta)$. 2

Theorem 3.1. We have:

$$
\operatorname{dim}\left(H^{i}\left(X, \mathcal{L}_{\infty}^{-1}\right)\right)=\left\{\begin{array}{l}
0, \quad i \neq \operatorname{dim}(\Delta) \\
N^{\circ}(\Delta), \quad i=\operatorname{dim}(\Delta)
\end{array}\right.
$$

We note that if $X$ is sufficiently complete for the polytope $\Delta=\Delta_{1}+\cdots+\Delta_{k}$ then it is also sufficiently complete for $m_{1} \Delta_{1}+\cdots+m_{k} \Delta_{k}$ for any integers $m_{i} \geq 0$. In particular, the cohomology groups of the line bundles associated to $m_{1} \Delta_{1}+\cdots+m_{k} \Delta_{k}$ are also given by Theorem 3.1

Corollary 3.2. Let $X$ be a sufficiently complete for $\Delta$. Then the Euler characteristic $\chi\left(X, \mathcal{L}_{\infty}^{-1}\right)$ is equal to $N^{\prime}(\Delta)$.

\footnotetext{
${ }^{2}$ In Khovanskii77 Khovanskii78 instead of our notation $N(\Delta), N^{\circ}(\Delta)$ and $N^{\prime}(\Delta)$ respectively the notation $T(\Delta), B^{+}(\Delta)$ and $B(\Delta)$ is used.
} 
Corollary 3.3. For any smooth projective toric variety $X$ we have $h^{0,0}(X)=1$ and $h^{i, 0}(X)=0$ for $i>0$.

Proof. The numbers $h^{i, 0}$ are birational invariants and any $n$-dimensional toric variety is birationaly equivalent to $\mathbb{C P}^{n}$. For $\mathbb{C P}^{n}$ the corollary is obvious. Nevertheless let us deduce the corollary from Theorem 3.1. Let $\Delta=\{0\}$ be the polytope consisting of the single point 0 . Then $\operatorname{dim}(\Delta)=0$ and $N^{\circ}(\Delta)=1$. Any smooth projective toric variety $X$ is sufficiently complete for $\Delta=\{0\}$ and the divisor $D_{\infty}$ on $X$ is $\{0\}$. Now apply Theorem 3.1 for $\Delta=\{0\}$.

We now use the results in Section 2.3 to give a condition for when a generic complete intersection $Z$ is irreducible. We also prove a result about the $h^{p, 0}$ numbers of $Z$.

For an integral polytope $\Delta \subset \mathbb{R}^{n}$ let $L_{\Delta}$ denote the linear subspace of Laurent polynomials in $\left(\mathbb{C}^{*}\right)^{n}$ spanned by all the monomials $x^{\alpha}$ where $\alpha \in \Delta \cap \mathbb{Z}^{n}$. It is easy to see that $L_{\Delta}$ has no base point on $\left(\mathbb{C}^{*}\right)^{n}$. Let $\Phi_{\Delta}:\left(\mathbb{C}^{*}\right)^{n} \rightarrow \mathbb{P}\left(L_{\Delta}^{*}\right)$ denote its Kodaira map. One observes that the dimension of the image of $\Phi_{\Delta}$ is equal to $\operatorname{dim}(\Delta)$.

As above, let $\Delta_{1}, \ldots, \Delta_{k}$ be integral polytopes in $\mathbb{R}^{n}$. For each $i=1, \ldots, k$ let $L_{i}=L_{\Delta_{i}}$ be the corresponding subspace of Laurent polynomials. From the above it follows that the defect of a subset $J \subset\{1, \ldots, k\}$ is equal to:

$$
d(J)=\operatorname{dim}\left(\Delta_{J}\right)-|J|,
$$

where $\Delta_{J}=\sum_{i \in J} \Delta_{i}$. We call $\Delta_{1}, \ldots, \Delta_{k}$ independent if the corresponding subspaces $L_{1}, \ldots, L_{k}$ are independent (see Definition 2.13). In other words, $\Delta_{1}, \ldots, \Delta_{k}$ are independent if $\operatorname{dim}\left(\sum_{i \in J} \Delta_{i}\right) \geq|J|$ for any subset $J \subset\{1, \ldots, k\}$.

Now let

$$
f_{1}(x)=\cdots=f_{k}(x)=0
$$

be a generic system of Laurent polynomials with Newton polytopes $\Delta_{1}, \ldots, \Delta_{k}$ respectively defining a complete intersection $Z$ in $\left(\mathbb{C}^{*}\right)^{n}$. We will assume that the polytopes $\Delta_{1}, \ldots, \Delta_{k}$ are independent. This guarantees that $Z$ is nonempty (Theorem 2.14). As before we let $X$ be a fixed smooth projective toric variety with $\left(\mathbb{C}^{*}\right)^{n}$ as the open orbit whose fan is a subdivision of the normal fan of the polytope $\Delta=\Delta_{1}+\cdots+\Delta_{k}$ (then $X$ is sufficiently complete with respect to the polytopes $\left.\Delta_{1}, \ldots, \Delta_{k}\right)$. Also let $X_{\infty}$ denote the sum of prime divisors in $X$ which lie in the complement of the open orbit $\left(\mathbb{C}^{*}\right)^{n}$. It is well-known that since $X$ is smooth, the divisor $X_{\infty}$ has normal crossings.

To apply the result in Sections 2.3 to the variety $Z$ we need the following lemma. For a proof see [Khovanskii77.

Lemma 3.4. With notation as above, let $D_{i}$ be the closure of the hypersurface defined by $f_{i}=0$ in $X$, for $i=1, \ldots, k$. Then $D_{i}$ is a smooth hypersurface and moreover all the divisors $D_{i}$ and the closures of all the $(n-1)$-dimensional orbits are mutually transverse in $X$.

Assume that the variety $Z$ is nonempty (i.e. the generic system (12) has solutions) and the conditions of Lemma 3.4 hold. Then the closure of $Z$ in $X$ is the intersection of the smooth divisors $D_{1}, \ldots, D_{k}$. As before, for each $i$ let $D_{i, \infty}$ denote the divisor at infinity on the toric variety $X$ associated to the polytope $\Delta_{i}$ and let $\mathcal{L}_{i, \infty}$ be its corresponding line bundle. Theorem 3.1 and the remark after it, give us the needed information about the dimensions of the cohomology groups $H^{i}\left(X, \mathcal{L}_{1, \infty}^{\otimes m_{1}} \otimes \cdots \otimes \mathcal{L}_{k, \infty}^{\otimes m_{k}}\right), m_{1}, \ldots, m_{k} \in\{0,-1\}$. 
Theorem 3.5. The arithmetic genus $\chi(Z)$ of $Z$, defined by a genreic system (12), is given by:

$$
\chi(X)=1-\sum_{i_{1}} N^{\prime}\left(\Delta_{i_{1}}\right)+\sum_{i_{1}<i_{2}} N^{\prime}\left(\Delta_{i_{1}}+\Delta_{i_{2}}\right)-\cdots+(-1)^{k} N^{\prime}\left(\Delta_{1}+\cdots+\Delta_{k}\right) .
$$

Recall that $N^{\prime}(\Delta)$ denotes $(-1)^{\operatorname{dim}(\Delta)} N^{\circ}(\Delta)$ and $N^{\circ}(\Delta)$ is the number of integral points in the interior of $\Delta$.

Proof. Theorem follows immediately from Theorem 2.20] and Corollary 3.2

Remark 3.6. For $k=n$ the righthand side of the (13) is equal to $n ! V\left(\Delta_{1}, \ldots, \Delta_{n}\right)$ where $V$ denotes the mixed volume of convex bodies ([Bernstein75]). So the Bernstein-Kushnirenko theorem follows from Theorem 3.5 (note that if $\operatorname{dim}(Z)=0$ then $\chi(Z)$ is equal to the number of points in $Z$ ). The formula for $\chi(Z)$ above and deduction of the Bernstein-Kushnerenko theorem from this formula are from Khovanskii78.

Finally we have the following theorems about the $h^{p, 0}$ numbers of the complete intersection $Z$ :

Theorem 3.7. For any integer $i \geq 0$ the following holds:

$$
h^{i, 0}(Z) \leq \sum_{\left\{J\left|\operatorname{dim}\left(\Delta_{J}\right)-\right| J \mid=i \text { and } J \neq \emptyset\right\}} N^{\circ}\left(\Delta_{J}\right)+\delta_{0}^{i},
$$

where $\delta_{0}^{i}=0$ for $i \neq 0$ and $\delta_{0}^{0}=1$.

Proof. The theorem follows from Theorem 2.21 Theorem 3.1 and the identity $h^{i, 0}(X)=\delta_{0}^{i}$ (see Corollary 3.3).

Definition 3.8. Let $\Delta_{1}, \ldots, \Delta_{k}$ be a $k$-tuple of independent integral polytopes. We say that a number $i \geq 0$ is critical for $\Delta_{1}, \ldots, \Delta_{k}$ if there is a nonempty set $J \subset\{1, \ldots, k\}$ such that $N^{\circ}\left(\Delta_{J}\right)>0$ and $\operatorname{dim}\left(\Delta_{J}\right)-|J|=i$.

Theorem 3.9. Let $\Delta_{1}, \ldots, \Delta_{k}$ be a $k$-tuple of independent integral polytopes.

(a) If 0 is a non-critical number for the collection of the $\Delta_{i}$ then the variety $Z$ defined by a generic system (12) is irreducible.

(b) If $i>0$ is a non-critical number for $\Delta_{1}, \ldots, \Delta_{k}$ then $h^{i, 0}(Z)=0$.

Proof. For a non-critical $i$ the inequality in Theorem 3.7 becomes $h^{i, 0}(Z) \leq \delta_{0}^{i}$. But the number $h^{0,0}(Z)$ is equal to the number of irreducible components of $Z$ and therefore it is strictly positive. The numbers $h^{i, 0}(Z)$ are nonnegative.

Theorem 3.7 implies the following improvement of a result in [Khovanskii78:

Corollary 3.10. If all the numbers $0 \leq i<n-k$ are not critical for $\Delta_{1}, \ldots, \Delta_{k}$ then $h^{0,0}(Z)=1, h^{n-k, 0}(Z)=(-1)^{(n-k)}(\chi(Z)-1)$ and $h^{p, 0}(Z)=0$ for $p \neq 0$ and $p \neq n-k$.

Proof. From Theorem 3.7 we have $h^{0,0}(Z)=1$ and $h^{p, 0}(Z)=0$ for $0<p<n-k$. The dimension of the smooth variety $Z$ is $n-k$ and therefore $h^{p, 0}(Z)=0$ for $n-k<p$.

\section{Virtual POLYTopes}

In this section we recall some basic facts from the theory of finitely additive measures on virtual polytopes developed in Khovanskii-Pukhlikov93. We will need them later in Section 5 . This theory extends the theory of valuations on convex polyhtopes due to Peter McMullen (McMullen77]) and uses the integration with respect to the Euler characteristic developed by Oleg Viro ([Viro88]). 
4.1. Ring $Z(\Lambda)$ of convex $\Lambda$-chains. For a fixed natural number $N$ let $\frac{1}{N} \mathbb{Z}^{n}$ be the lattice:

$$
\frac{1}{N} \mathbb{Z}^{n}=\left\{a / N \mid a \in \mathbb{Z}^{n}\right\} \subset \mathbb{R}^{n} .
$$

In the rest of this section $\Lambda \subset \mathbb{R}^{n}$ denotes an additive subgroup of $\mathbb{R}^{n}$ which is either equal to $\mathbb{R}^{n}$ itself or is equal to $\frac{1}{N} \mathbb{Z}^{n}$ for some natural number $N$. We denote by $\mathcal{P}(\Lambda)$ the collection of all convex polytopes in $\mathbb{R}^{n}$ with vertices in $\Lambda$. We call the elements of $\mathcal{P}(\Lambda)$ the $\Lambda$-polytopes. When $\Lambda=\mathbb{R}^{n}$ (respectively $\Lambda=\mathbb{Z}^{n}$ ) we refer to the $\Lambda$-polytopes simply as polytopes (respectively integral polytopes).

Definition 4.1. A convex $\Lambda$-chain (or a $\Lambda$-chain for short) is a function $\alpha: \mathbb{R}^{n} \rightarrow \mathbb{Z}$ which can be represented as a finite sum $\alpha=\sum_{i} n_{i} \chi_{\Delta_{i}}$, where $\chi_{Y}$ denotes the characteristic function of a set $Y$, the $\Delta_{i}$ are convex polytopes in $\mathcal{P}(\Lambda)$ and $n_{i} \in \mathbb{Z}$. We denote the additive group of convex $\Lambda$-chains by $Z(\Lambda)$.

Let $\Delta^{\circ}$ be the set of interior points of a polyhedron $\Delta$, in the topology of the affine space spanned by $\Delta$. It is easy to see that

$$
\chi_{\Delta^{\circ}}=\sum_{\Delta_{i} \in \Gamma(\Delta)}(-1)^{\operatorname{dim} \Delta_{i}} \chi_{\Delta_{i}},
$$

where $\Gamma(\Delta)$ is the set of all faces of $\Delta$, including $\Delta$ itself. Therefore if $\Delta$ is in $\mathcal{P}(\Lambda)$ then $\chi_{\Delta} \circ$ is also in $Z(\Lambda)$.

It is shown in Khovanskii-Pukhlikov93 that the Minkowski sum of convex polytopes extends in a unique way to an opration $*$ :

$$
*: Z(\Lambda) \times Z(\Lambda) \rightarrow Z(\Lambda),
$$

on the additive group of $\Lambda$-convex chains. That is, for two convex polytopes $\Delta_{1}, \Delta_{2} \in \mathcal{P}(\Lambda)$ we have:

$$
\chi_{\Delta_{1}} * \chi_{\Delta_{2}}=\chi_{\left(\Delta_{1}+\Delta_{2}\right)} .
$$

We call the operation $*$ the (Minkowski) multiplication of chains. The group $Z(\Lambda)$ together with this multiplication is a commutative ring called the ring of $\Lambda$-convex chains.

The characteristic function of the origin $\chi_{\{0\}}$ is the unit in the ring $Z(\Lambda)$. We call any invertible element of the ring of $\Lambda$-chains a $\Lambda$-virtual polytope. When $\Lambda=\mathbb{R}^{n}$ (respectively $\Lambda=\mathbb{Z}^{n}$ ) we simply call an invertible element a virtual polytope (respectively an integral virtual polytope). It turns out that the characteristic function $\chi_{\Delta}$ of a polytope $\Delta \in \mathcal{P}(\Lambda)$ is invertible in the $\operatorname{ring} Z(\Lambda)$ :

Theorem 4.2 ( $\Lambda$-virtual polytopes). The following statements hold:

(1) For $\Delta \in \mathcal{P}(\Lambda)$ the multiplicative inverse $\chi_{\Delta}^{-1}$ of $\chi_{\Delta} \in Z(\Lambda)$ is

$$
(-1)^{\operatorname{dim} \Delta} \chi_{\left(-\Delta^{\circ}\right)}
$$

Here $-\Delta^{\circ}$ is the set of interior points of $-\Delta$.

(2) Each $\Lambda$-virtual polytope can be written in the form $\chi_{\Delta_{1}} * \chi_{\Delta_{2}}^{-1}$, where $\Delta_{1}, \Delta_{2} \in \mathcal{P}(\Lambda)$.

4.2. Integration over the Euler characteristic and multiplication in the $\operatorname{ring} Z(\Lambda)$. There is a way to define the multiplication $*$ in the ring of convex chains $Z(\Lambda)$ which does not require representation of convex chains as linear combinations of characteristic functions of polytopes. This definition instead uses integration with respect to the Euler characteristic (see Viro88). Below we explain this notion in more detail. 
Let us say that a subset $Y$ in a finite dimensional real vector space $V$ is semi-convex if $Y$ can be represented as a disjoint union

$$
Y=\bigcup_{i} \Delta_{i}^{\circ}
$$

of relative interiors of finitely many convex polytopes $\Delta_{i} \in \mathcal{P}(\Lambda)$. By definition the Euler characteristic $\mu(Y)$ of a semi-convex set $Y$ is $\sum_{i}(-1)^{\operatorname{dim} \Delta_{i}}$. It is known (see [Viro88]) that $\mu(Y)$ is well-defined, i.e. is independent of a choice of the representation (14). The Euler characteristic is a finitely additive measure on the collection of semi-convex sets. For closed semi-convex sets this measure coincides with the usual Euler characteristic in topology, but for a general semi-convex set it is not the topological Euler characteristic. One defines the integral $\int f d \mu$ of a convex chain $f \in Z\left(\mathbb{R}^{n}\right)$ with respect to the Euler characteristic by:

$$
\int f d \mu=\sum_{a \in \mathbb{Z}} a \mu\left(f^{-1}(a)\right) .
$$

(Note that $f$ takes only finitely many values and that each level set $f^{-1}(a)$ is a semi-convex set.)

Theorem 4.3 (Multiplication in $Z(\Lambda)$ using the Euler characteristic). The multiplication $\alpha * \beta$ of $\alpha, \beta \in Z(\Lambda)$ is equal to the convolution with respect to the integration over Euler characteristic of the functions $\alpha, \beta$ :

$$
(\alpha * \beta)(x)=\int \alpha(z) \beta(x-z) d \mu(z) .
$$

4.3. Finitely additive measures on convex $\Lambda$-chains. A (real valued finitely additive) measure on $Z(\Lambda)$ is by definition an additive homomorphism $\phi: Z(\Lambda) \rightarrow \mathbb{R}$. A measure is called an invariant measure (respectively a polynomial measure of degree $\leq d$ ) if for each $\alpha \in Z(\Lambda)$ the function $h \mapsto \phi\left(\alpha_{h}\right)$ on $h \in \Lambda$ is constant (respectively is the restriction of a polynomial of degree $\leq d$ to $\Lambda \subset \mathbb{R}^{n}$ ). Here $\alpha_{h} \in Z(\Lambda)$ is the convex chain defined by $\alpha_{h}(x)=\alpha(x-h)$ (i.e. $\alpha_{h}$ is the shift of $\alpha$ by $h$ ).

One can check the following easy facts:

Lemma 4.4. Let $\phi: Z\left(\mathbb{R}^{n}\right) \rightarrow \mathbb{R}$ be a measure. Then:

(1) For $\beta \in Z\left(\mathbb{R}^{n}\right)$ the function $\phi_{\beta}: Z\left(\mathbb{R}^{n}\right) \rightarrow \mathbb{R}$ defined by $\phi_{\beta}(\alpha)=\phi(\alpha * \beta)$ is also a measure on $Z\left(\mathbb{R}^{n}\right)$.

(2) The restriction of $\phi$ to $Z(\Lambda) \subset Z\left(\mathbb{R}^{n}\right)$ is a measure on $Z(\Lambda)$.

Lemma 4.5. Let $f: \mathbb{R}^{n} \rightarrow \mathbb{R}$ be a polynomial of degree $\leq d$. Then the function $\phi=\int f$ on $Z\left(\mathbb{R}^{n}\right)$ defined by $\phi(\alpha)=\int f(x) \alpha(x) d x$, where $d x$ is the standard Lebesgue measure on $\mathbb{R}^{n}$, is a polynomial measure of degree $\leq d$ on $Z\left(\mathbb{R}^{n}\right)$.

In particular, Lemma 4.5 states that the map $\Delta \mapsto \int_{\Delta} f(x) d x$ on the space of polytopes $\mathcal{P}\left(\mathbb{R}^{n}\right)$ uniquely extends to a measure on virtual polytopes. This measure is the usual volume of a polytope if $f$ is the constant polynomial 1 .

Lemma 4.6. Let $f: \mathbb{R}^{n} \rightarrow \mathbb{R}$ be a polynomial of degree $\leq d$ and let $\beta \in Z\left(\mathbb{R}^{n}\right)$ be a convex chain. Then the function $\phi$ on integral convex chains $Z\left(\mathbb{Z}^{n}\right)$ defined by:

$$
\phi(\alpha)=\sum_{x \in \mathbb{Z}^{n}} f(x)(\beta * \alpha)(x),
$$

is a polynomial measure of degree $\leq d$ on integral convex chains. 
In particular Lemma 4.6 implies the following: let $\Delta_{\beta}$ be a fixed (not necessarily integral) convex polytope. Consider the map $\phi: \Delta \mapsto \sum_{x \in\left(\Delta+\Delta_{\beta}\right) \cap \mathbb{Z}^{n}} f(x)$ on convex polytopes. Then this map extends uniquely to a measure on virtual convex polytopes. If $f$ is the constant polynomial 1 then $\phi(\Delta)$ is the number of integral points in $\Delta+\Delta_{\beta}$.

We now state the main statement about polynomial measures on virtual polytopes. Fix a $k$-tuple of $\Lambda$-virtual polytopes $\alpha_{1}, \ldots, \alpha_{k} \in Z(\Lambda)$ as well as a polynomial measure $\phi$ of degree $\leq d$ on $Z(\Lambda)$. Let $\Phi$ be the function on $\mathbb{Z}^{k}$ defined by:

$$
\Phi\left(m_{1}, \ldots, m_{k}\right)=\phi\left(\alpha_{1}^{m_{1}} * \cdots * \alpha^{m_{k}}\right),
$$

where $\left(m_{1}, \ldots, m_{k}\right) \in \mathbb{Z}^{k}$ and $\alpha_{i}^{m_{i}}$ denotes the $m_{i}$-th power of $\alpha_{i}$ in the $\operatorname{ring} Z(\Lambda)$.

Theorem 4.7. With the above notation, the function $\Phi$ is the restriction of a polynomial of degree $\leq n+d$ on $\mathbb{R}^{k}$ to $\mathbb{Z}^{k}$.

The next corollary follows from Theorem 4.7 applied to the measure in Lemma 4.6 .

Corollary 4.8. Let $f: \mathbb{R}^{n} \rightarrow \mathbb{R}$ be a degree $d$ polynomial, $\beta$ a convex chain and $\alpha_{1}, \ldots, \alpha_{k}$ a $k$-tuple of integral virtual polytopes. Then the function $\Phi$ on the lattice $\mathbb{Z}^{k}$ defined by:

$$
\Phi\left(m_{1}, \ldots, m_{k}\right)=\sum_{x \in \mathbb{Z}^{n}} f(x)\left(\beta * \alpha_{1}^{m_{1}} * \cdots * \alpha_{k}^{m_{k}}\right)(x),
$$

where $\left(m_{1}, \ldots, m_{k}\right) \in \mathbb{Z}^{k}$, is the restriction of a polynomial on $\mathbb{R}^{k}$ of degree $\leq n+d$ to $\mathbb{Z}^{k}$.

Finally we have the following variation of Corollary 4.8. We will use it later in Section 5.5 and Section 5.8. Below $\Lambda=\frac{1}{N} \mathbb{Z}^{n} \subset \mathbb{R}^{n}$ for some ineteger $N>0$.

Corollary 4.9. Let $f: \mathbb{R}^{n} \rightarrow \mathbb{R}$ be a polynomial of degree $d$ and let $\gamma_{1}, \ldots, \gamma_{k} \in \mathcal{P}(\Lambda)$ be a $k$-tuple of $\Lambda$-virtual polytopes. Let $a_{1}, \ldots, a_{k}$ be fixed points in the lattice $\Lambda$ and consider the function $\Psi: \mathbb{Z}^{k} \rightarrow \mathbb{R}$ defined by:

$$
\Psi\left(m_{1}, \ldots, m_{k}\right)=\sum_{x \in\left(m_{1} a_{1}+\cdots+m_{k} a_{k}\right)+\mathbb{Z}^{n}} f(x)\left(\gamma_{1}^{m_{1}} * \cdots * \gamma_{k}^{m_{k}}\right)(x) .
$$

Then $\Psi$ is the restriction of a polynomial on $\mathbb{R}^{k}$ to $\mathbb{Z}^{k}$.

Proof. We reduce the claim to Corollary 4.8. Consider the space $\mathbb{R}^{n+k}=\mathbb{R}^{n} \times \mathbb{R}^{k}$, the lattice $\Lambda_{1}=\frac{1}{N} \mathbb{Z}^{n+k} \subset \mathbb{R}^{n+k}$ and the $\Lambda_{1}$-chains $\rho_{1}, \ldots, \rho_{k}$ defined by $\rho_{i}=\gamma_{i} * \delta_{\left(-a_{i}, e_{i}\right)}$. Here $e_{i}$ is the $i$-th standard basis vector in $\mathbb{R}^{k}$ and $\delta_{(a, b)}$ denotes the characteristic function of a point $(a, b) \in \mathbb{R}^{n} \times \mathbb{R}^{k}$. Then $\rho_{1}^{m_{1}} * \cdots * \rho_{k}^{m_{k}}=\gamma_{1}^{m_{1}} * \cdots * \gamma_{k}^{m_{k}} * \delta_{\left(-m_{1} a_{1}-\cdots-m_{k} a_{k}, m_{1} e_{1}+\cdots+m_{k} e_{k}\right)}$. Also consider the polynomial $h: \mathbb{R}^{n} \times \mathbb{R}^{k} \rightarrow \mathbb{R}$ defined by: $h\left(x, m_{1}, \ldots, m_{k}\right)=f\left(x+a_{1} m_{1}+\right.$ $\left.\cdots+a_{k} m_{k}\right)$. The claim now follows from Corollary 4.8 applied to the vector space $\mathbb{R}^{n+k}$, the lattice $\Lambda_{1}=\frac{1}{N} \mathbb{Z}^{n+k} \subset \mathbb{R}^{n+k}, \Lambda_{1}$-chains $\rho_{1}, \ldots, \rho_{k}$ and the polynomial $h$ (we take $\beta$ in Corollary 4.8 to be 0 ).

\section{Complete intersections in SPhericAl VARIETIES}

In the rest of the paper we will use the following notation about reductive groups:

- $G$ denotes a connected complex reductive algebraic group.

- $B$ a Borel subgroup of $G$ and $T, U$ the maximal torus and maximal unipotent subgroups contained in $B$ respectively.

- $\Lambda$ is the weight lattice of $G, \Lambda^{+}$is the subset of dominant weights and $\Lambda_{\mathbb{R}}=\Lambda \otimes_{\mathbb{Z}} \mathbb{R}$. The cone generated by $\Lambda^{+}$is the positive Weyl chamber denoted by $\Lambda_{\mathbb{R}}^{+}$. 
- $V_{\lambda}$ denotes the irreducible $G$-module corresponding to a dominant weight $\lambda$. Also $v_{\lambda}$ denotes a highest weight vector in $V_{\lambda}$.

- $G / H$ denotes a spherical homogeneous space.

- $\Lambda^{\prime}=\Lambda(G / H) \subset \Lambda$ denotes the weight lattice of $G / H$, i.e. the sublattice of $\Lambda$ consisting of weights of $B$-eigenfucntions in $\mathbb{C}(G / H)$.

5.1. Preliminaries on spherical varieties. A $G$-variety $X$ is called spherical if a Borel subgroup (and hence any Borel subgroup) has a dense orbit. If $X$ is spherical it has a finite number of $G$-orbits as well as a finite number of $B$-orbits. Spherical varieties are a generalization of toric varieties for actions of reductive groups. Analogous to toric varieties, the geometry of spherical varieties can be read off from associated convex polytopes and convex cones. For a nice overview of the theory of spherical varieties we refer the reader to Perrin14.

It is a well-known fact that if $\mathcal{L}$ is a $G$-linearized line bundle on a spherical variety then the space of sections $H^{0}(X, \mathcal{L})$ is a multiplicity free $G$-module. For a quasi-projective $G$-variety $X$ this is actually equivalent to $X$ being spherical.

Below are some important examples of spherical varieites and spherical homogeneous spaces:

(1) When $G$ is a torus, the spherical $G$-varieties are exactly toric varieties.

(2) The flag variety $G / B$ and the parital flag varieties $G / P$ are spherical $G$-varieties by the Bruhat decomposition.

(3) Let $G \times G$ act on $G$ from left and right. Then the stabilizer of the identity is $G_{\text {diag }}=\{(g, g) \mid g \in G\}$. Thus $G$ can be identified with the homogeneous space $(G \times G) / G_{\text {diag }}$. Again by the Bruhat decomposition this is a spherical $(G \times G)$ homogeneous space.

(4) Consider the set $\mathcal{Q}$ of all smooth quadrics in $\mathbb{P}^{n}$. The group $G=\operatorname{PGL}(n+1, \mathbb{C})$ acts transitively on $\mathcal{Q}$. The stabilizer of the quadric $x_{0}^{2}+\cdots+x_{n}^{2}=0$ (in the homogeneous coordinates) is $H=\mathrm{PO}(n+1, \mathbb{C})$ and hence $\mathcal{Q}$ can be identified with the homogeneous space $\operatorname{PGL}(n+1, \mathbb{C}) / \mathrm{PO}(n+1, \mathbb{C})$. The subgroup $\mathrm{PO}(n+1, \mathbb{C})$ is the fixed point set of the involution $g \mapsto\left(g^{t}\right)^{-1}$ of $G$ and hence $\mathcal{Q}$ is a symmetric homogeneous space. In particular, $\mathcal{Q}$ is spherical. Let $V$ be the vector space of all quadratic forms in $n+1$ variables and $V^{*}$ its dual. The map which assigns to a quadric $C$ its homogeneous equation (respectively equation of the dual quadric $C^{*}$ ) gives an embedding of $\mathcal{Q}$ in $\mathbb{P}(V)$ (respectively $\mathbb{P}\left(V^{*}\right)$ ). Let $X$ be the closure of the set of all quadrics $\left(C, C^{*}\right)$ in $\mathbb{P}(V) \times \mathbb{P}\left(V^{*}\right)$. It is called the variety of complete quadrics. It is well-known that $X$ is a smooth variety (see [DeConcini-Procesi82, Theorem 3.1]). This variety plays an important role in classical enumerative geometry.

Throughout the rest of the paper we will fix a spherical homogeneous space $G / H$.

Definition 5.1. Let $\Lambda(G / H)$ be the lattice of $B$-weights for the action of $B$ on the field of rational functions $\mathbb{C}(G / H)$, i.e. the set of all $\lambda \in \Lambda$ which appear as the weight of a $B$ eigenfunction in $\mathbb{C}(G / H)$. Clearly $\Lambda(G / H)$ is a sublattice of $\Lambda$. We will denote the lattice of $B$-weights of $G / H$ simply by $\Lambda^{\prime}$.

Remark 5.2. Let $\mathbb{C}(G / H)^{(B)}$ denote the multiplicative group of nonzero $B$-eigenfunctions in $\mathbb{C}(G / H)$. If two $B$-eigenfunctions $f$ and $g$ have the same weight then $f / g$ is a $B$-invariant rational function on $G / H$. Since $X$ has an open $B$-orbit we conclude that $f / g$ is constant. This proves that the map which sends a $B$-eigenfunction to its weight gives an isomorphism between $\mathbb{C}(G / H)^{(B)} / \mathbb{C}^{*}$ and the lattice $\Lambda^{\prime}$. 
The following theorem about $h^{p, 0}$ numbers of spherical varieties will be used later. It is a generalization of Corollary 3.3 for toric varieties.

Theorem 5.3 ( $h^{p, 0}$ numbers of spherical varieties). Let $X$ be a smooth projective spherical variety. Then $h^{0,0}(X)=1$ and $h^{p, 0}(X)=0$ if $p>0$.

Proof. One shows that if $X$ is a spherical variety then the maximal torus $T$ has isolated fixed points. The theorem then follows from the more general theorem that if $X$ is a smooth projective variety with an action of a torus with isolated fixed points then $h^{p, q}(X)=0$ if $p \neq$ $q$. We refer to Carrell-Liebermann73 for this vanishing result as well as its generalization to holomorphic vector fields on Kähler manifolds.

5.2. Cohomology of line bundles. Let $X$ be an $n$-dimensional projective spherical variety with a globally generated $G$-linearized line bundle $\mathcal{L}$. The following result of Michel Brion determines when the cohomology groups of the line bundles $\mathcal{L}$ and $\mathcal{L}^{-1}$ vanish ([Brion90]). It generalizes similar statements for toric varieties as well as the Borel-Weil-Bott theorem for flag varieites. Below $\kappa=\kappa(\mathcal{L})$ denotes the Itaka dimension of $\mathcal{L}$, i.e. the dimension of the image of the Kodaira map of $\mathcal{L}^{\otimes m}$ for sufficiently large $m$.

Theorem 5.4 (Brion). Let $X$ be a projective spherical variety with a globally generated $G$-linearized line bundle $\mathcal{L}$. Then:

(a) For any $i>0, H^{i}(X, \mathcal{L})=\{0\}$.

(b) For all $i \neq \kappa, H^{i}\left(X, \mathcal{L}^{-1}\right)=\{0\}$.

As before we let $\chi(X, \mathcal{L})$ denote the Euler characteristic of the bundle $\mathcal{L}$ defined by $\chi(X, \mathcal{L})=\sum_{i=0}^{n}(-1)^{i} \operatorname{dim}\left(H^{i}(X, \mathcal{L})\right)$.

Corollary 5.5. Let $X$ be a smooth projective spherical variety and $\mathcal{L}_{1}, \ldots, \mathcal{L}_{k}$ globally generated $G$-linearized line bundles on $X$. For integers $m_{1}, \ldots, m_{k}$ put:

$$
\psi\left(m_{1}, \ldots, m_{k}\right)=\chi\left(X, \mathcal{L}_{1}^{\otimes m_{1}} \otimes \cdots \otimes \mathcal{L}_{k}^{\otimes m_{k}}\right)
$$

Then $\psi$ is a polynomial in the $m_{i}$. Moreover when the $m_{i}$ are nonnegative we have:

$$
\psi\left(m_{1}, \ldots, m_{k}\right)=\operatorname{dim}\left(H^{0}\left(X, \mathcal{L}_{1}^{\otimes m_{1}} \otimes \cdots \otimes \mathcal{L}_{k}^{\otimes m_{k}}\right)\right) .
$$

Proof. The corollary follows from the Hirzbruch-Riemann-Roch theorem and Theorem[5.4(a).

In particular, let $\mathcal{L}$ be a globally generated $G$-linearized line bundle on a projective spherical variety $X$. Let $\psi$ be the polynomial in $m \in \mathbb{Z}$ such that $\psi(m)=\operatorname{dim}\left(H^{0}\left(X, \mathcal{L}^{\otimes m}\right)\right)$ for any nonnegative integer $m$.

Corollary 5.6. As above let $\kappa=\kappa(\mathcal{L})$ be the Itaka dimension of $\mathcal{L}$. We can compute the dimension of $H^{\kappa}\left(X, \mathcal{L}^{-1}\right)$ by:

$$
\operatorname{dim}\left(H^{\kappa}\left(X, \mathcal{L}^{-1}\right)\right)=(-1)^{\kappa} \psi(-1) .
$$

(Note that by Theorem 5.4(b) all other cohomology groups of $\mathcal{L}^{-1}$ are 0. )

Proof. The corollary follows from the Hirzbruch-Riemann-Roch theorem and Theorem 5.4(b). 
5.3. Moment polytope. In this section we discuss the notion of the moment polytope $\Delta(R)$ of a graded $G$-algebra $R=\bigoplus_{m} R_{m}$. When $R$ is the homogeneous coordinate ring of a smooth projective $G$-variety (equivariantly embedded in a projective space) the moment polytope can be identified with the moment polytope (or Kirwan polytope) of $X$ in symplectic geometry (see Remark 5.8 below). We will be interested in the moment polytopes of graded $G$-linear systems $R$ over a spherical variety $X$. When $X$ is normal, the integral points in the dilated polytope $m \Delta(R)$ correspond to the irreducible $G$-modules $V_{\lambda}$ appearing in $R_{m}$ (Theorem [5.9). We will express our main formula for the genus of a complete intersection in terms of integral points in moment polytopes (Theorem 5.29).

5.3.1. Moment polytope of a graded $G$-algebra. Let $R=\bigoplus_{m} R_{m}$ be a graded $G$-algebra over $\mathbb{C}$ where the $R_{m}$ are finite dimensional. Moreover, assume that $R$ is contained in a finitely generated graded algebra. The rings $R$ we will be interested in the upcoming sections are $G$-invariant graded linear systems on a spherical variety or a homogeneous space. That is, graded subalgebras of rings of sections of $G$-line bundles on a spherical variety.

Consider the additive semigroup $S(R) \subset \mathbb{N} \times \Lambda$ defined by:

$$
S(R)=\bigcup_{m}\left\{(m, \lambda) \mid V_{\lambda} \text { appears in } R_{m}\right\} .
$$

Let $C(R)$ denote the closure of the convex hull of $S(R)$ in the vector space $\mathbb{R} \times \Lambda_{\mathbb{R}}$. It is a closed convex cone with apex at the origin. One defines the moment convex body $\Delta(A)$ to be the slice of the cone $C(R)$ at $m=1$ (Brion87] and Kaveh-Khovanskii12b]). That is:

$$
\Delta(R)=C(R) \cap\left(\{1\} \times \Lambda_{\mathbb{R}}\right) .
$$

Alternatively, after projection $\mathbb{R} \times \Lambda_{\mathbb{R}} \rightarrow \Lambda_{\mathbb{R}}$ on the second factor, the polytope $\Delta(R)$ can be defined as:

$$
\Delta(R)=\overline{\bigcup_{m>0}\left\{\lambda / m \mid V_{\lambda} \text { appears in } R_{m}\right\}} .
$$

Remark 5.7. If the algebra $R$ is finitely generated then one shows that the semigroup $S(R)$ is a finitely generated semigroup and hence $\Delta(R)$ is a rational convex polytope. In this case we will refer to $\Delta(R)$ as the moment polytope. The moment polytope is also called the Brion polytope. It was first introduced in the paper [Brion87.

Remark 5.8 (Connection with moment polytope in symplectic geometry). Let $K$ be a compact Lie group and let $X$ be a compact Hamiltonian $K$-manifold with the moment map $\phi: X \rightarrow \operatorname{Lie}(K)^{*}$. It is a well-known result due to $\mathrm{F}$. Kirwan that the intersection of the image of the moment map with the positive Weyl chamber is a convex polytope usually called the moment polytope or Kirwan polytope of the Hamiltonian $K$-space $X$.

As usual let $G$ be a complex connected reductive group. Let $G$ act linearly on a finite dimensional complex vector space $V$. Let $X$ be a closed irreducible $G$-stable subvariety of the projective space $\mathbb{P}(V)$. Let $R$ denote the homogenous coordinate ring of $X$. Fix a $K$ invariant inner product on $V$ where $K$ is a maximal compact subgroup of $G$. This induces a $K$-invariant symplectic structure on $\mathbb{P}(V)$ and hence on the smooth locus of $X$.

(1) With this symplectic structure, the smooth locus of $X$ is a Hamiltonian $K$-manifold.

(2) When $X$ is smooth it can be proved that the Kirwan polytope of $X$ coincides with $\Delta(R)$ (see Ness84, Guillemin-Sternberg84 and Brion87). More precisely, the Kirwan polytope identifies with $\Delta(R)$ after taking the involution $\lambda \mapsto \lambda^{*}$, where $\lambda^{*}=-w_{0} \lambda$. 
(3) The above is still true if $X$ is non-smooth. In this case one considers the moment map of $\mathbb{P}(V)$ (as a Hamiltonian $K$-space) and restricts it to $X$. Then the intersection of the image of $X$ (under the restricted moment map) with the positive Weyl chamber can be identified with $\Delta(R)$.

5.3.2. Moment polytope of a line bundle on a spherical variety. In this section we describe the linear inequalities defining the moment polytope of the ring of sections of a $G$-linearized line bundle on a normal projective spherical variety. We will use it to describe the space of sections of the line bundle, as a $G$-module, in terms of the lattice points in its moment polytope.

Let $\mathcal{L}$ be a $G$-linearized line bundle on a normal projective spherical $G$-variety $X$. Then $H^{0}(X, \mathcal{L})$ is a finite dimensional $G$-module. Let us assume that $H^{0}(G, \mathcal{L}) \neq\{0\}$. By the ring of sections of $\mathcal{L}$ we mean the algebra $R(X, \mathcal{L})$ defined by:

$$
R(X, \mathcal{L})=\bigoplus_{m} H^{0}\left(X, \mathcal{L}^{\otimes m}\right) .
$$

We denote the moment convex body of this algebra by $\Delta(X, \mathcal{L})$.

Since $H^{0}(X, \mathcal{L})$ is a finite dimensional $G$-module there is a $B$-eigensection $\sigma$ in $H^{0}(X, \mathcal{L})$. Then the divisor $D$ of the section $\sigma$ is a $B$-stable divisor. Let $D_{1}, \ldots, D_{s}$ be all the $B$-stable prime divisors in $X$. Thus we can write:

$$
D=\sum_{i} a_{i} D_{i}
$$

Then for a rational function $f \in \mathbb{C}(X)$ the corresponding mermorphic section $f \sigma$ belongs to $H^{0}(X, \mathcal{L})$ if and only if it satisfies:

$$
\operatorname{ord}_{D_{i}}(f) \geq-a_{i}, \quad \forall i=1, \ldots, s,
$$

where $\operatorname{ord}_{D_{i}}$ is the order of zero-pole along the prime divisor $D_{i}$ (notice that here we are using the assumption that $X$ is normal). Via restriction, the function $\operatorname{ord}_{D_{i}}$ now defines a linear function $\ell_{D_{i}}$ on the lattice $\Lambda^{\prime}=\Lambda(G / H) \cong \mathbb{C}(G / B)^{(B)} / \mathbb{C}^{*}$ (see Remark 5.2). Let $\alpha$ denote the weight of the $B$-eigensection $\sigma \in H^{0}(X, \mathcal{L})$ that we fixed. Then the $G$-spectrum of $H^{0}(X, \mathcal{L})$ can be described as:

$$
\begin{aligned}
& \operatorname{Spec}_{G}\left(H^{0}(X, \mathcal{L})\right)=\alpha+\left\{\gamma \in \Lambda^{\prime} \mid \ell_{D_{i}}(\gamma) \geq-a_{i}, 1 \leq i \leq s\right\} \\
& =\left\{\lambda \in \alpha+\Lambda^{\prime} \mid \ell_{D_{i}}(\lambda) \geq-a_{i}+\ell_{D_{i}}(\alpha), 1 \leq i \leq s\right\} .
\end{aligned}
$$

Applying this to all the $H^{0}\left(X, \mathcal{L}^{\otimes m}\right)$ for $m>0$, we get the following description of the moment polytope of the ring of sections $R(X, \mathcal{L})$ :

$$
\Delta(X, \mathcal{L})=\left\{x \in \alpha+\Lambda_{\mathbb{R}}^{\prime} \mid \ell_{D_{i}}(x) \geq-a_{i}+\ell_{D_{i}}(\alpha), 1 \leq i \leq s\right\} .
$$

$\left(\Lambda_{\mathbb{R}}^{\prime}\right.$ is the vector space spanned by the lattice $\Lambda^{\prime}$.)

Conversely, suppose $\lambda=\alpha+\gamma \in \alpha+\Lambda^{\prime}$ is a shifted lattice point which lies in the polytope $\Delta(X, \mathcal{L})$. By (16) for any $i=1, \ldots, s$ we have $\ell_{D_{i}}(\gamma) \geq-a_{i}$. And hence if $f$ is a $B$-eigenfunction with weight $\gamma$ then for any $i$ we have $\operatorname{ord}_{D_{i}}(f) \geq-a_{i}$ which implies that $f \sigma \in H^{0}(X, \mathcal{L})$. Thus we have proved:

Theorem 5.9 (Brion).

$$
H^{0}(X, \mathcal{L})=\bigoplus_{\lambda \in \Delta(X, \mathcal{L}) \cap\left(\alpha+\Lambda^{\prime}\right)} V_{\lambda}
$$


Theorem 5.9 immediately gives us the dimension of the space of sections $H^{0}(X, \mathcal{L})$ :

$$
\operatorname{dim}\left(H^{0}(X, \mathcal{L})\right)=\sum_{\lambda \in \Delta(X, \mathcal{L}) \cap\left(\alpha+\Lambda^{\prime}\right)} \operatorname{dim}\left(V_{\lambda}\right)
$$

For a rational polytope $\Delta \subset \Lambda_{\mathbb{R}}$ and $a \in \Lambda$ we define the number $S(\Delta, a)$ by:

$$
S(\Delta, a)=\sum_{\lambda \in \Delta \cap\left(a+\Lambda^{\prime}\right)} f(\lambda)
$$

where $f$ is the Weyl polynomial given by the formula:

$$
f(\lambda)=\operatorname{dim}\left(V_{\lambda}\right)=\prod_{\alpha \in \Phi^{+}}\langle\lambda+\rho, \alpha\rangle /\langle\rho, \alpha\rangle .
$$

By the Weyl dimension formula, for any dominant weight $\lambda$, the dimension of $V_{\lambda}$ is equal to $f(\lambda)$. With this notation in place, we can restate (17) as:

Proposition 5.10. For any interger $m>0$ we have:

$$
\operatorname{dim}\left(H^{0}\left(X, \mathcal{L}^{\otimes m}\right)\right)=S(m \Delta(X, \mathcal{L}), m \alpha) .
$$

Next we consider the line bundle $\mathcal{L}^{-1}$. To describe the cohomology group $H^{\kappa}\left(X, \mathcal{L}^{-1}\right)$, where $\kappa=\kappa(\mathcal{L})$, we need some more notation. Let $\Delta$ be a rational polytope in $\Lambda_{\mathbb{R}}$ and $a \in \Lambda$. By Corollary 4.9, the function: $\Psi(m)=S(m \Delta, m a)$ is a polynomial. We put $S^{\circ}(\Delta, a)$ to be $(-1)^{\operatorname{dim}(\Delta)+d_{\Delta}} \Psi(-1)$, where $d_{\Delta}$ is the degree of the Weyl polynomial $f$ restricted to the affine span of $\Delta$ in $\Lambda_{\mathbb{R}}$. In the light of Theorem $4.2(1), S^{\circ}(\Delta, a)$ can also be defined as:

$$
S^{\circ}(\Delta, a)=(-1)^{d_{\Delta}} \sum_{\lambda \in \Delta^{\circ} \cap\left(a+\Lambda^{\prime}\right)} f(-\lambda) .
$$

As usual $\Delta^{\circ}$ denotes the interior of $\Delta$ (in the topology of the affine span of $\Delta$ ).

The following theorem determines the dimension of the cohomology group $H^{\kappa}(X, \mathcal{L})$ where $\kappa=\kappa(\mathcal{L})$ is the Itaka dimension. We note that by Theorem 5.9 the Itaka dimension $\kappa=\kappa(\mathcal{L})$ is equal to $\operatorname{dim}(\Delta(X, \mathcal{L}))+d_{\Delta(X, \mathcal{L})}$.

Theorem 5.11. $\operatorname{dim}\left(H^{\kappa}\left(X, \mathcal{L}^{-1}\right)\right)=S^{\circ}(\Delta(X, \mathcal{L}), \alpha)$.

Proof. By Corollary 4.9 we know that $\Psi(m)=S(m \Delta(X, \mathcal{L}), m \alpha)$ is a polynomial in $m$. Moreover, by Corollary 5.5 we know that for any nonnegative integer $m$ :

$$
\chi\left(X, \mathcal{L}^{\otimes m}\right)=\operatorname{dim}\left(H^{0}\left(X, \mathcal{L}^{\otimes m}\right)\right)=S(m \Delta(X, \mathcal{L}), m \alpha),
$$

where $\chi\left(X, \mathcal{L}^{\otimes m}\right)$ denotes the Euler characteristic of the bundle $\mathcal{L}^{\otimes m}$. Putting these together we conclude that $\operatorname{dim}\left(H^{\kappa}\left(X, \mathcal{L}^{-1}\right)\right)=(-1)^{\kappa} \Psi(-1)$. Note that the polynomial $\chi\left(X, \mathcal{L}^{\otimes m}\right)$ has degree $\kappa$ which is equal to $\operatorname{dim}(\Delta)+d_{\Delta}$. This finishes the proof.

5.4. Newton-Okounkov polytope. Fix a reduced decomposition $\underline{w}_{0}$ for the longest element $w_{0}$ in the Weyl group of $G$. To any $\lambda$ in the positive Weyl chamber $\Lambda_{\mathbb{R}}^{+}$one can associate a convex rational polytope $\Delta_{\underline{w}_{0}}(\lambda) \subset \mathbb{R}^{N}$ called the string polytope associated to $\lambda$ (and $\underline{w}_{0}$ ). Here $N$ is the number of positive roots. The polytope $\Delta_{\underline{w}_{0}}(\lambda)$ has the property that, when $\lambda$ is a dominant weight, the number of integral points in $\vec{\Delta}_{\underline{w}_{0}}(\lambda)$ is equal to the dimension of the irreducible $G$-module $V_{\lambda}$, i.e.:

$$
\operatorname{dim}\left(V_{\lambda}\right)=\#\left(\Delta_{\underline{w}_{0}}(\lambda) \cap \mathbb{Z}^{N}\right) .
$$

In fact, the integral points in $\Delta_{\underline{w}_{0}}(\lambda)$ are in one-to-one correspondence with the so-called canonical basis for $V_{\lambda}$ ([Littelmann98, Berenstein-Zelevinsky01]). 
The string polytopes generalize the well-known Gelfand-Zetlin polytopes $\Delta_{\mathrm{GZ}}(\lambda)$ associated to irreducible representations of $\mathrm{GL}(n, \mathbb{C})$ (Gelfand-Zetlin50). That is, for a specific choice of a reduced decomposition, namely $w_{0}=\left(s_{1}\right)\left(s_{2} s_{1}\right)\left(s_{3} s_{2} s_{1}\right)\left(s_{n} s_{n-1} \cdots s_{1}\right)$, for the longest element of the Weyl group of $\operatorname{GL}(n, \mathbb{C})$ the string polytopes can be identified with the Gelfand-Zetlin polytopes.

Remark 5.12. The dependence of $\Delta_{\underline{w}_{0}}(\lambda)$ is piecewise linear, in the sense that there is a rational polyhedral cone $C_{\underline{w}_{0}}$ (with apex at the origin) in the vector space $\mathbb{R}^{N} \times \Lambda_{\mathbb{R}}$ such that for each $\lambda \in \Lambda_{\mathbb{R}}^{+}$the string polytope $\Delta_{\underline{w}_{0}}(\lambda)$ is the slice of the cone $C_{\underline{w}_{0}}$ at $\lambda$, i.e. $\Delta_{\underline{w}_{0}}(\lambda)=C_{\underline{w}_{0}} \cap \pi^{-1}(\lambda)$ where $\pi: \mathbb{R}^{N} \times \Lambda_{\mathbb{R}} \rightarrow \Lambda_{\mathbb{R}}$ is the projection on the second factor. Note that we can then define $\Delta_{\underline{w}_{0}}(\lambda)$ for all $\lambda \in \Lambda_{\mathbb{R}}$ (not just integral $\lambda$ ).

Moreover, when $G=\operatorname{GL}(n, \mathbb{C})$ it is easy to see from the defining inequalities of the Gelfand-Zetlin polytopes that $\lambda \mapsto \Delta_{\mathrm{GZ}}(\lambda)$ is additive, namely for any $\lambda, \gamma \in \Lambda_{\mathbb{R}}^{+}$we have:

$$
\Delta_{\mathrm{GZ}}(\lambda+\gamma)=\Delta_{\mathrm{GZ}}(\lambda)+\Delta_{\mathrm{GZ}}(\gamma) .
$$

Now let $X$ be a spherical $G$-variety with a $G$-linearized line bundle $\mathcal{L}$. Let $R=\bigoplus_{m} R_{m}$ be a graded $G$-subalgebra of the ring of sections $\bigoplus_{m} H^{0}\left(X, \mathcal{L}^{m}\right)$. Moreover assume that $R_{m}$ is finite dimensional for all $m$ (this is automatic if $X$ is projective). We call $R$ a graded $G$-linear system. We would like to associate a polytope $\tilde{\Delta}(R)$ to $R$ which is responsible for the dimensions of the homogeneous pieces $R_{m}$.

Definition 5.13. Let $\tilde{\Delta}(R)$ denote the Newton-Okounkov polytope of $R$, that is, the polytope over $\Delta(R)$ with string polytopes $\Delta_{\underline{w}_{0}}(\lambda)$ as fibers:

$$
\tilde{\Delta}(R)=\bigcup_{\lambda \in \Delta(R)}\left(\{\lambda\} \times \Delta_{\underline{w}_{0}}(\lambda)\right) \subset \Lambda_{\mathbb{R}}^{+} \times \mathbb{R}^{N} .
$$

If $\mathcal{L}$ is a $G$-linearized line bundle on a projective spherical $G$-variety $X$ we denote the Newton-Okounkov polytope of the ring of sections $R(X, \mathcal{L})$ by $\tilde{\Delta}(X, \mathcal{L})$.

Remark 5.14. The above notion of the Newton-Okounkov polytope of a graded $G$-linear system over a spherical variety is a special case of the more general notion of a NewtonOkounkov body of a graded linear system on an arbitrary variety (see Lazarsfeld-Mustata09, Kaveh-Khovanskii12a and the references therein).

Let $\mathcal{L}$ be a $G$-linearized line bundle on a normal projective spherical $G$-variety $X$ which contains $G / H$ as the open $G$-orbit. As usual $\Lambda^{\prime}=\Lambda(G / H)$ denotes the lattice of weights of $B$-eigenfunctions in $\mathbb{C}(G / H)$. Let us assume that $H^{0}(X, \mathcal{L}) \neq\{0\}$ and fix a weight $\alpha$ of a $B$-eigensection in $H^{0}(X, \mathcal{L})$.

Let $\tilde{\Delta}$ be a rational polytope in $\mathbb{R}^{n}$. Fix a lattice $L \subset \mathbb{R}^{n}$ and a point $a \in \mathbb{R}^{n}$. We denote by $N(\tilde{\Delta}, a)$ (respectively $\left.N^{\circ}(\tilde{\Delta}, a)\right)$ the number of points in the shifter lattice $a+L$ which lie in $\tilde{\Delta}$ (respectively in the interior of $\tilde{\Delta}$ in the topology of its affine span). We note that, by Theorem $4.2(1), N^{\circ}(\tilde{\Delta}, a)$ is equal to the $(-1)^{\operatorname{dim}(\tilde{\Delta})}$ times the value of the polynomial $m \mapsto N(m \tilde{\Delta}, m a)$ at $m=-1$. These notations slightly extend $N$ and $N^{\circ}$ defined in Section 3.

Proposition 5.15 (Newton-Okounkov polytope and dimension of space of sections). Fix the lattice $\Lambda^{\prime} \times \mathbb{Z}^{N}$ in the (real) vector space $\Lambda_{\mathbb{R}} \times \mathbb{R}^{N}$. Consider $\alpha \in \Lambda$ as an element of $\Lambda \times \mathbb{Z}^{N}$. Then for any integer $m>0$ we have:

$$
\operatorname{dim}\left(H^{0}\left(X, \mathcal{L}^{\otimes m}\right)\right)=N(m \tilde{\Delta}(X, \mathcal{L}), m \alpha) .
$$


Moreover:

$$
\operatorname{dim}\left(H^{\kappa}\left(X, \mathcal{L}^{-1}\right)\right)=N^{\circ}(\tilde{\Delta}(X, \mathcal{L}), \alpha),
$$

where as before $\kappa=\kappa(\mathcal{L})$ is the Itaka dimension.

Proof. The proposition follows from Proposition 5.10 and Theorem [5.11.

5.5. Euler characteristic of line bundles over a projective spherical variety. Let $X$ be a projective spherical $G$-variety. In this section we consider the cases where the moment polytope or the Newton-Okounkov polytope are additive. In these cases, extending the case of toric varieties, the formula for Euler characteristic of line bundles on $X$ can be expressed as polynomial measures on convex chains (Section 4.3).

Let $\mathcal{L}_{1}, \ldots, \mathcal{L}_{k}$ be $G$-linearized line bundles over a projective spherical variety $X$. We assume that the bundles are globally generated (i.e. the $H^{0}\left(X, L_{i}\right)$ are base point free linear systems on $X)$. Consider the bundle $\mathcal{L}_{1}^{\otimes m_{1}} \otimes \cdots \otimes \mathcal{L}_{k}^{\otimes m_{k}}$. Consider any collection of non negative numbers $m_{1}, \ldots, m_{k}$, such that at least one number is not zero. Below we will assume that one the following two conditions on these bundles hold:

(I) The Newton-Okounkov polytope $\tilde{\Delta}$ of the bundle $\mathcal{L}_{1}^{\otimes m_{1}} \otimes \cdots \otimes \mathcal{L}_{k}^{\otimes m_{k}}$ is equal to the Minkowski sum $\tilde{\Delta}_{1}+\cdots+\tilde{\Delta}_{k}$ where $\tilde{\Delta}_{1}, \ldots, \tilde{\Delta}_{k}$ are the Newton-Okounkov polytope of the bundles $\mathcal{L}_{1}, \ldots, \mathcal{L}_{k}$ respectively.

(II) The moment polytope $\Delta$ of the bundle $\mathcal{L}_{1}^{\otimes m_{1}} \otimes \cdots \otimes \mathcal{L}_{k}^{\otimes m_{k}}$ is equal to the Minkowski sum $\Delta_{1}+\cdots+\Delta_{k}$ where $\Delta_{1}, \ldots, \Delta_{k}$ are the moment polytopes of the bundles $\mathcal{L}_{1}, \ldots, \mathcal{L}_{k}$ respectively.

Remark 5.16. (1) For $k=1$ The Conditions I and II always hold but when $k>1$ they do not always hold.

(2) The condition (II) above holds in the classes of horospherical homogeneous spaces as well as the group case (see Sections 6.1 and 6.2). The condition (I) holds in these two classes of examples provided that the string polytopes are also additive. This for example happens for the well-known Gelfand-Zetlin polytopes (which are a special case of the string polytopes for $G=\mathrm{GL}(n, \mathbb{C})$ ).

Let as usual $\Lambda^{\prime}$ denotes weight lattice of $X$, i.e. the sublattice of the weight lattice $\Lambda$ consisting of all the weights of $B$-eigenfunctions on $X$. For each $i=1, \ldots, k$ fix a weight $a_{i} \in \Lambda$ of a $B$-eigensection in $H^{0}\left(X, \mathcal{L}_{i}\right)$.

Theorem 5.17. Assume that for the bundles $\mathcal{L}_{1}, \ldots, \mathcal{L}_{k}$ the condition (I) holds. Then for any $k$-tuple of integral numbers $m_{1}, \ldots, m_{k}$ the Euler characteristic of $X$ with the coefficients in the bundle $\mathcal{L}_{1}^{\otimes m_{1}} \otimes \cdots \otimes \mathcal{L}_{k}^{\otimes m_{k}}$ is given by:

$$
\chi\left(X, \mathcal{L}_{1}^{\otimes m_{1}} \otimes \cdots \otimes \mathcal{L}_{k}^{\otimes m_{k}}\right)=\sum_{x \in\left(m_{1} a_{1}+\cdots+m_{k} a_{k}\right)+\left(\Lambda^{\prime} \times \mathbb{Z}^{N}\right)} \tilde{\gamma}_{1}^{m_{1}} * \cdots * \tilde{\gamma}_{k}^{m_{k}},
$$

where $\tilde{\gamma}_{i}$ is the characteristic function of the Newton-Okounkov polytope $\tilde{\Delta}\left(X, \mathcal{L}_{i}\right)$.

Proof. By the Hirzbruch-Riemann-Roch theorem the Euler characteristic is a polynomial in the $m_{1}, \ldots, m_{k}$. Moreover by Proposition [5.15 it coincides with the righthand side of (23) for $m_{i} \geq 0$. On the other hand, by Corollary 4.9 the righthand side of (23) is also a polynomial in $m_{1}, \ldots, m_{k}$. Thus the two polynomials must coincide.

Theorem 5.18. Assume that for the bundles $\mathcal{L}_{1}, \ldots, \mathcal{L}_{k}$ the condition (II) holds. Then for any $k$-tuple of integral numbers $m_{1}, \ldots, m_{k}$ the Euler characteristic of $X$ with the coefficients 
in the bundle $\mathcal{L}_{1}^{\otimes m_{1}} \otimes \cdots \otimes \mathcal{L}_{k}^{\otimes m_{k}}$ is given by:

$$
\chi\left(X, \mathcal{L}_{1}^{\otimes m_{1}} \otimes \cdots \otimes \mathcal{L}_{k}^{\otimes m_{k}}\right)=\sum_{x \in\left(m_{1} a_{1}+\cdots+m_{k} a_{k}\right)+\Lambda^{\prime}} f(x) \gamma_{1}^{m_{1}} * \cdots * \gamma_{k}^{m_{k}},
$$

where $\gamma_{i}$ is the characteristic function of the moment polytope $\Delta\left(X, \mathcal{L}_{i}\right)$ and $f$ is the Weyl polynomial (see (19)).

Proof. By the Hirzbruch-Riemann-Roch theorem the Euler characteristic is a polynomial in the $m_{1}, \ldots, m_{k}$. Also by Proposition 5.10 it coincides with the righthand side of (24) for $m_{i} \geq 0$. Again by Corollary 4.9 the righthand side is also polynomial and the two polynomials must coincide.

5.6. Invariant linear systems on a spherical homogeneous space. In this section we discuss invariant linear systems on a spherical homogenous space $G / H$ and their associated moment polytopes. First we consider the case of invariant subspaces of regular functions and then the general case of invariant linear systems.

5.6.1. Moment polytope of an invariant subspace of regular functions. Let us assume that the homogeneous space $G / H$ is quasi-affine. In this section we consider the case of trivial line bundles, i.e. we have finite dimensional subspaces of regular functions on $G / H$. This situation is closer to the classical toric case and Newton polytope theory which is concerned with finite dimensional subspaces of Laurent polynomials spanned by monomials.

Example 5.19. (a) Let $H=U$ be a maximal unipotent subgroup of $G$. One can show that $G / U$ is quasi-affine and as a $G$-module (for the left $G$-action on $G / U$ ) the space of regular functions $\mathbb{C}[G / U]$ decomposes as:

$$
\mathbb{C}[G / U]=\bigoplus_{\lambda \in \Lambda^{+}} V_{\lambda}
$$

(b) Consider the left-right action of $G \times G$ on $G$. Then $G \cong(G \times G) / G_{\text {diag }}$ is affine and the space of regular functions on $G$, as a $(G \times G)$-module, decomposes as:

$$
\mathbb{C}[G]=\bigoplus_{\lambda \in \Lambda^{+}} \operatorname{End}\left(V_{\lambda}\right)
$$

As usual let $\Lambda^{\prime}=\Lambda(G / H) \subset \Lambda$ be the lattice of weights of $B$-eigenfunction in $\mathbb{C}(G / H)$. Also let $\Lambda^{\prime+}=\Lambda^{+}(G / H)$ denote the weights of the $B$-eigenfunctions in the algebra $\mathbb{C}[G / H]$. It is clear that $\Lambda^{\prime+}$ is a semigroup in $\Lambda$. One knows that the $G$-algebra $\mathbb{C}[G / H]$ decomposes into finite dimensional irreducible $G$-modules. Thus every $B$-eigenfunction in $\mathbb{C}[G / H]$ is actually a highest weight vector for $G$ and generates an irreducible $G$-module. Thus:

$$
\Lambda^{\prime+}=\left\{\lambda \in \Lambda^{+} \mid V_{\lambda} \text { appear in } \mathbb{C}[G / H]\right\} .
$$

The following is well-known (see Timashev06]):

Proposition 5.20. The semigroup $\Lambda^{\prime+}$ generates the lattice $\Lambda^{\prime}$.

Now let $\mathcal{A} \subset \Lambda^{\prime+}$ be a finite subset. To $\mathcal{A}$ there corresponds the finite dimensional $G$-invariant subspace:

$$
L_{\mathcal{A}}=\bigoplus_{\lambda \in \mathcal{A}} V_{\lambda} \subset \mathbb{C}[G / H]
$$

Since $L_{\mathcal{A}}$ is $G$-invariant it is automatically base point free and hence its Kodaira map is defined everywhere on $G / H$. Let $\Phi_{\mathcal{A}}: G / H \rightarrow \mathbb{P}\left(L_{\mathcal{A}}^{*}\right)$ denote the Kodaira map of the $G$-invariant subspace $L_{\mathcal{A}}$. It is a $G$-equivariant morphism. Also let $Y_{\mathcal{A}}$ denote the closure 
of the image of $G / H$ in the projective space $\mathbb{P}\left(L_{\mathcal{A}}^{*}\right)$. We note that the dimension of the projective variety $Y_{\mathcal{A}}$ could possibly be smaller than that of $G / H$.

Let $\overline{R(\mathcal{A})}$ be the integral closure of the algebra $R(\mathcal{A})=\bigoplus_{m} L_{\mathcal{A}}^{m}$ in $\bigoplus_{m} \mathbb{C}(G / H)$. We know that:

$$
\overline{R(\mathcal{A})}=\bigoplus_{m} \overline{L_{\mathcal{A}}^{m}}
$$

where $\bar{L}$ denotes the integral closure (or completion) of a subspace $L$ in the field of rational functions $\mathbb{C}(G / H)$ (see Samuel-Zariski60, Appendix 4]). We denote the moment polytope of this graded algebra by $\Delta(\mathcal{A})$. It is a convex polytope containing $\mathcal{A}$.

We have the following description of the $G$-modules $\overline{L_{\mathcal{A}}^{m}}$ in terms of the moment polytope $\Delta(\mathcal{A})$ :

Theorem 5.21. For every integer $m>0$, the $G$-module $\overline{L_{\mathcal{A}}^{m}}$ decomposes as:

$$
\overline{L_{\mathcal{A}}^{m}}=\bigoplus_{\lambda \in m \Delta(\mathcal{A}) \cap \Lambda^{\prime}} V_{\lambda} .
$$

Proof. As in the proof of Theorem [5.24, given the $G$-invariant subspace $L_{\mathcal{A}}$ we can find a normal projective spherical $G$-variety $X$ which contains $G / H$ as the open orbit and moreover the Kodaira map $\Phi_{\mathcal{A}}$ extends to the whole $X$. Let $\mathcal{L}=\Phi_{\mathcal{A}}^{*}(\mathcal{O}(1))$ be the pull-back of the line bundle $\mathcal{O}(1)$ on the projective space to $X$. One shows that for each $m>0$ the integral closure $\overline{L_{\mathcal{A}}^{m}}$ can be identified with $H^{0}\left(X, \mathcal{L}^{\otimes m}\right)$. The theorem now follows from Theorem 5.9. Note that $\alpha$ is the identity character because $\mathbb{C}[G / H]$ contains the constant function 1 which is invariant under the action of $G$ and hence is a $B$-eigensection with weight 0 .

5.6.2. Moment polytope of an invariant linear system. Let $\mathcal{E}$ be a $G$-linearized line bundle on $G / H$. Then the space of sections $H^{0}(G / H, \mathcal{E})$ is a $G$-module. Moreover, since $G / H$ is spherical, $H^{0}(G / H, \mathcal{E})$ is multiplicity-free, that is, every irreducible $G$-module appears in it with multiplicity 0 or 1 .

Take a finite nonempty subset $\mathcal{A}$ of the $G$-spectrum of the space of global sections $H^{0}(G / H, \mathcal{E})$. Let $E_{\mathcal{A}}$ denote the $G$-invariant subspace of $H^{0}(G / H, \mathcal{E})$ determined by $\mathcal{A}$, that is:

$$
E_{\mathcal{A}}=\bigoplus_{\lambda \in \mathcal{A}} V_{\lambda}
$$

Since $E_{\mathcal{A}}$ is $G$-invariant then the base locus of $E_{\mathcal{A}}$, i.e. the locus of points where all the sections in $E_{\mathcal{A}}$ vanish, is a $G$-invariant subvariety of $G / H$. But as $E_{\mathcal{A}}$ is nonzero we conclude that the base locus of $E_{\mathcal{A}}$ is empty, in other words, $E_{\mathcal{A}}$ is base point free. The base point free linear system $E_{\mathcal{A}}$ gives rise to a Kodaira map $\Phi_{\mathcal{A}}: X \rightarrow \mathbb{P}\left(E_{\mathcal{A}}^{*}\right)$, the projective space of the dual space $E_{\mathcal{A}}^{*}$. We denote the closure of the image of the Kodaira map by $Y_{\mathcal{A}}$. The Kodaira map is $G$-equivariant and hence $Y_{\mathcal{A}}$ is a $G$-stable subvariety of the projective space $\mathbb{P}\left(E_{\mathcal{A}}^{*}\right)$. Note that the dimension of the projective variety $Y_{\mathcal{A}}$ may be smaller than that of $X$. To $\mathcal{A}$ we can associate a graded algebra:

$$
R(\mathcal{A})=\bigoplus_{m} E_{\mathcal{A}}^{m}
$$

where $E_{\mathcal{A}}^{m}$ denotes the image of $E_{\mathcal{A}} \otimes \cdots \otimes E_{\mathcal{A}}(m$ times $)$ in $H^{0}\left(X, \mathcal{E}^{\otimes m}\right)$ under the product map $H^{0}(X, \mathcal{E}) \otimes \cdots \otimes H^{0}(X, \mathcal{E})(m$ times). The algebra $R(\mathcal{A})$ is a graded $G$-subalgebra of the ring of sections $\bigoplus_{m} H^{0}\left(X, \mathcal{E}^{\otimes m}\right)$. The homogneous coordinate ring of the projective variey $Y_{\mathcal{A}} \subset \mathbb{P}\left(E_{\mathcal{A}}^{*}\right)$ can naturally be identified with $R(\mathcal{A})$. 
Let $\mathbb{C}(\mathcal{E})$ denote the space of meromorphic sections of $\mathcal{E}$. It can be identified with $\mathbb{C}(G / H)$ via a choice of a nonzero section $\sigma \in \mathbb{C}(\mathcal{E})$. We denote the integral closure of the algebra $R(\mathcal{A})$ in $\bigoplus_{m} \mathbb{C}\left(\mathcal{E}^{\otimes m}\right)$ by $\overline{R(\mathcal{A})}$.

We have the following extension of Theorem 5.21

Theorem 5.22. For every integer $m>0$, the $G$-module $\overline{E_{\mathcal{A}}^{m}}$ decomposes as:

$$
\overline{E_{\mathcal{A}}^{m}}=\bigoplus_{\lambda \in m \Delta(\mathcal{A}) \cap\left(m \alpha+\Lambda^{\prime}\right)} V_{\lambda}
$$

Here $\alpha$ denotes the weight of a B-eigensection $\sigma$ in $H^{0}(G / H, \mathcal{E})$.

Proof. As in the proof of Theorem 5.21 .

5.7. Simultaneous resolution of singularities. Let $\mathcal{E}_{1}, \ldots, \mathcal{E}_{k}$ be globally generated $G$ linearized line bundles on a spherical homogeneous space $G / H$. For each $i=1, \ldots, k$ let $E_{i}$ be a nonzero $G$-invariant linear system for $\mathcal{E}_{i}$ i.e. $E_{i}$ is a finite dimensional $G$-invariant subspace of $H^{0}\left(X, \mathcal{E}_{i}\right)$. Each $E_{i}$ is $G$-invariant and hence it is base point free. Thus the Kodaira map $\Phi_{E_{i}}$ is defined on the whole $G / H$. As usual we denote the closure of the image of the Kodaira map $\Phi_{E_{i}}$ by $Y_{E_{i}}$. It is a projective $G$-subvariety of $\mathbb{P}\left(E_{i}^{*}\right)$.

We will be interested in a generic complete intersection of $E_{1}, \ldots, E_{k}$ in $G / H$. To apply topological methods, we need to work with a compactification of $G / H$ which behaves nicely with respects to the linear systems $E_{i}$. This is the content of the next definition. It is an extension of the similar notion for toric varieties ([Khovanskii77]).

Definition 5.23. Let $X$ be a $G$-equivariant completion of $G / H$, that is, $X$ is a complete spherical $G$-variety which contains $G / H$ as the open orbit. Let us say that $X$ is sufficiently complete with respect to the linear systems $E_{1}, \ldots, E_{k}$ if:

(a) $X$ is smooth.

(b) For each $i=1, \ldots, k$, the Kodaira map $\Phi_{E_{i}}$ extends to a morphism on the whole $X$.

Theorem 5.24. Given base point free $G$-invariant linear systems $E_{1}, \ldots, E_{k}$ on $G / H$ there exists a projective spherical variety $X$ which is sufficiently complete with respect to $E_{1}, \ldots, E_{k}$.

Proof. One knows that the homogeneous space $G / H$ has an equivariant projective completion. Let $Z$ be such a projective completion, i.e. $Z$ is a projective spherical $G$-variety which contains $G / H$ as the open orbit. Consider the map $\Phi: G / H \rightarrow Z \times Y_{E_{1}} \times \cdots \times Y_{E_{k}}$ given by:

$$
\Phi(x)=\left(x, \Phi_{E_{1}}(x), \ldots, \Phi_{E_{k}}(x)\right),
$$

and let $Y$ be the closure of the image of $\Phi$. The map $\Phi$ is a $G$-equivariant embedding and $Y$ is a projective subvariety of $Z \times Y_{E_{1}} \times \cdots \times Y_{E_{k}}$. We note that $Y$ contains $G / H$ as an open orbit and hence is a spherical $G$-variety. We have the following commutative diagram:

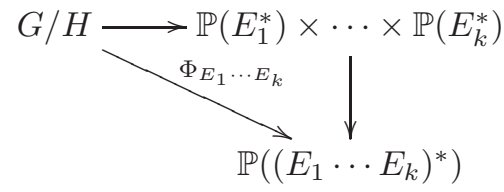

where the vertical arrow is the Segre map and $E_{1} \ldots E_{k}$ is the linear system which is the image of $E_{1} \otimes \cdots \otimes E_{k}$ in $H^{0}\left(G / H, \mathcal{E}_{1} \otimes \cdots \otimes \mathcal{E}_{k}\right)$. Thus in defining $Y$, instead of the Kodaira maps $\Phi_{E_{1}}, \ldots, \Phi_{E_{k}}$, we alternatively could use the Kodaira map $\Phi_{E_{1} \cdots E_{k}}$. 
The following theorem guarantees that $Y$ has a $G$-equivariant resolution of singularities (see Perrin14).

Theorem 5.25 (Resolution of singularities for spherical varieties). Every spherical $G$ variety has a G-equivariant resolution of singularities.

Let $\pi: X \rightarrow Y$ be a $G$-equivariant resolution of singularities of $Y$ as in the above theorem. For each $i$ let $\pi_{i}$ be the projection $Z \times Y_{E_{1}} \times \cdots \times Y_{E_{k}} \rightarrow Y_{E_{i}}$ restricted to $Y$. For each $i=1, \ldots, k$ we have a commutative diagram:

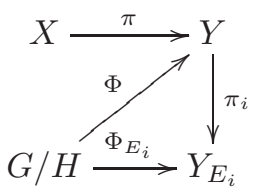

Note that $\Phi$ (respectively $\pi$ ) is an isomorphisms from $G / H$ (respectively the open orbit in $X)$ to the open orbit in $Y$. Let us identify the homogeneous space $G / H$ with the open orbit in $X$ via $\pi^{-1} \circ \Phi$. Now for each $i$ define $\tilde{\Phi}_{E_{i}}$ to be $\pi_{i} \circ \pi$. Clearly $\tilde{\Phi}_{E_{i}}$ is $G$-equivariant and extends the Kodaira map $\Phi_{E_{i}}: G / H \rightarrow Y_{E_{i}}$. This finishes the proof of the theorem.

The following is a direct corollary of Thom's transversality theorem (Theorem 2.9):

Theorem 5.26 (Transversality of generic hyperplane sections). As above let $E_{1}, \ldots, E_{k}$ be $G$-invariant nonzero linear systems on a spherical homogeneous space $G / H$ and $X$ a sufficiently complete completion of $G / H$ with respect to the $E_{i}$. For $f_{i} \in E_{i}$ let $H_{i}=$ $\overline{\left\{x \in G / H \mid f_{i}(x)=0\right\}} \subset X$ be the closure of the hypersurface defines by $f_{i}$. Let $f_{i} \in E_{i}$ be generic. We then have:

(1) For each $i$ the hypersurface $H_{i} \subset X$ is smooth.

(2) For each $i$ the hypersurace $H_{i}$ intersects all the $G$-orbits in $X$ transversely.

(3) Either the intersection of the hypersurfaces $H_{1}, \ldots, H_{k}$ is empty or they intersect transversely.

5.8. Genus and $h^{p, 0}$ numbers of a complete intersection. As in Section 5.7 let $\mathcal{E}_{1}, \ldots, \mathcal{E}_{k}$ be a $G$-linearized line bundle on $G / H$. For each $i=1, \ldots, k$ fix a dominant weight $\alpha_{i}$ for a $B$-eigensection in $H^{0}\left(G / H, \mathcal{E}_{i}\right)$. Also for each $i=1, \ldots, k$ let $\mathcal{A}_{i}$ be a finite subset of $\operatorname{Spec}_{G}\left(H^{0}\left(G / H, \mathcal{E}_{i}\right)\right)$ and:

$$
E_{i}=\bigoplus_{\lambda \in \mathcal{A}_{i}} V_{\lambda} \subset H^{0}\left(G / H, \mathcal{E}_{i}\right),
$$

the corresponding $G$-invariant linear system. Also we denoted by $\Delta\left(E_{i}\right)$ the moment polytope of the $G$-algebra $\overline{\bigoplus_{m} E_{i}^{m}}$.

By Theorem 5.24 we can find a sufficiently complete projective completion $X$ of $G / H$ with respect to $E_{1}, \ldots, E_{k}$. For each $i=1, \ldots, k$ let $\mathcal{L}_{i}=\Phi_{E_{i}}^{*}(\mathcal{O}(1))$ be the pull-back of the line bundle $\mathcal{O}(1)$ on the projective space $\mathbb{P}\left(E_{i}^{*}\right)$ to $X$. The line bundle $\mathcal{L}_{i}$ is globally generated because it is the pull-back of a globally generated line bundle.

One shows that for any $m>0$ the integral closure $\overline{E_{i}^{m}}$ can be identified with the space of sections $H^{0}\left(X, \mathcal{L}_{i}^{\otimes m}\right)$. Hence the moment polytope $\Delta\left(X, \mathcal{L}_{i}\right)$ coincides with $\Delta\left(E_{i}\right)$, that is the moment polytope of the integral closure $\overline{\bigoplus_{m} E_{i}^{m}}$ (in particular the moment polytope $\Delta\left(X, \mathcal{L}_{i}\right)$ is independent of the sufficiently complete completion $\left.X\right)$.

The next theorem gives a necessary and sufficient condition for a generic complete intersection $X_{k}$ from $E_{1}, \ldots, E_{k}$ to be nonempty, in terms of the dimensions of the NewtonOkounkov or moment polytopes. It is a direct corollary of Theorems 2.14 and 2.19. 
Theorem 5.27. A generic complete intersection $X_{k}$ from $E_{1}, \ldots, E_{k}$ is nonempty if and only if $E_{1}, \ldots, E_{k}$ are independent. That is, for any $J \subset\{1, \ldots, k\}$ we have $\operatorname{dim}\left(\tilde{\Delta}_{J}\right) \geq|J|$ (equivalently $\operatorname{dim}\left(\Delta_{J}\right)+d_{J} \geq|J|$ ). Here $\tilde{\Delta}_{J}$ (respectively $\Delta_{J}$ ) is the Newton-Okounkov polytope (respectively the moment polytope) of the linear system $E_{J}=\prod_{i \in J} E_{i}$ and $d_{J}$ is the degree of the Weyl polynomial restricted to the affine span of $\Delta_{J}$.

For the rest of the paper we assume that the condition in Theorem 5.27 is satisfied and hence a generic complete intersection $X_{k}$ in nonempty.

Let us recall some notation. As in Proposition 5.15 fix the lattice $\Lambda^{\prime} \times \mathbb{Z}^{N}$ in the vector space $\Lambda_{\mathbb{R}} \times \mathbb{R}^{N}$. Recall that for a rational polytope $\tilde{\Delta} \subset \Lambda_{\mathbb{R}} \times \mathbb{R}^{N}$ and a point $a \in \Lambda_{\mathbb{R}} \times \mathbb{Z}^{N}$, we denote by $N(\tilde{\Delta}, a)$ (respectively $N^{\circ}(\tilde{\Delta}, a)$ ) the number of points in the shifted lattice $a+\left(\Lambda^{\prime} \times \mathbb{Z}^{N}\right)$ which lie in $\tilde{\Delta}$ (respectively in the interior of $\left.\tilde{\Delta}\right)$. Moreover, we define $N^{\prime}(\tilde{\Delta}, a)$ to be:

$$
N^{\prime}(\tilde{\Delta}, a)=(-1)^{\operatorname{dim}(\tilde{\Delta})} N^{\circ}(\tilde{\Delta}, a) .
$$

In fact, $N^{\prime}(\tilde{\Delta}, a)$ is the value of the polynomial $m \mapsto N(m \tilde{\Delta}, m a)$ at $m=-1$. Similarly if $\Delta$ is a rational polytope in the vector space $\Lambda_{\mathbb{R}}$ and $\alpha \in \Lambda$ a weight, we denote by $S(\Delta, \alpha)$ the sum of values of the Weyl polynomial $f$ on the shifted lattice points $\alpha+\Lambda^{\prime}$ which lie in $\Delta$. Moreover, we denote by $S^{\prime}(\Delta, \alpha)$ the value of the polynomial $m \mapsto S(m \Delta, m \alpha)$ at $m=-1$. Using the notation introduced in the paragraph before Theorem [5.11 we can write:

$$
S^{\prime}(\Delta, \alpha)=(-1)^{\operatorname{dim}(\Delta)+d_{\Delta}} S^{\circ}(\Delta, \alpha) .
$$

Here $S^{\circ}(\Delta, \alpha)$ is $(-1)^{d \Delta}$ times the sum of values of the polynomial $f(-\lambda)$ on the shifted lattice points $\alpha+\Lambda^{\prime}$ which lie in the interior of $\Delta$.

Also recall that for each $i=1, \ldots, k, \alpha_{i} \in \Lambda$ is the weight of a $B$-eigensection in $E_{i}$. The moment polytope $\Delta\left(E_{i}\right)$ lies in the affine subspace $\alpha_{i}+\Lambda_{\mathbb{R}}^{\prime}$. We also consider $\alpha_{i}$ as $\left(\alpha_{i}, 0\right)$ in the larger lattice $\Lambda \times \mathbb{Z}^{N}$. The Newton-Okounkov polytope $\tilde{\Delta}\left(E_{i}\right)$ lies in the affine subspace $\alpha_{i}+\left(\Lambda_{\mathbb{R}}^{\prime} \times \mathbb{R}^{N}\right)$.

For each $i$, let $\kappa_{i}$ be the Itaka dimension of the linear system $E_{i}$. From Proposition 5.10 and Theorem 5.11, applied to the line bundles $\mathcal{L}_{i}$ on $X$, we obtain the following:

Theorem 5.28. With notation as above we have:

(a)

$$
\operatorname{dim}\left(H^{0}\left(X, \mathcal{L}_{i}\right)\right)=S\left(\Delta\left(E_{i}\right), \alpha_{i}\right)=N\left(\tilde{\Delta}\left(E_{i}\right), \alpha_{i}\right) .
$$

$$
\operatorname{dim}\left(H^{\kappa_{i}}\left(X, \mathcal{L}_{i}^{-1}\right)\right)=S^{\circ}\left(\Delta\left(E_{i}\right), \alpha_{i}\right)=N^{\circ}\left(\tilde{\Delta}\left(E_{i}\right), \alpha_{i}\right) .
$$

In particular, the cohomology groups of $\left(X, \mathcal{L}_{i}\right)$ and $\left(X, \mathcal{L}_{i}^{-1}\right)$ only depend on the $E_{i}$, i.e. are independent of the choice of the sufficiently complete completion $X$ for $G / H$.

We now use the above to compute the genus of a complete intersection from $E_{1}, \ldots, E_{k}$ in $G / H$. Let $f_{1}, \ldots, f_{k}$ be generic elements in $E_{1}, \ldots, E_{k}$ respectively. For each $i=1, \ldots, k$ let $D_{i}=\left\{x \in G / H \mid f_{i}(x)=0\right\}$ be the hypersurface defined by $f_{i}$ and let $X_{k}=D_{1} \cap \cdots \cap D_{k}$.

The next theorem is one of our main results.

Theorem 5.29 (Genus of a complete intersection in a spherical homogenous space). With notation as above we have:

$$
\begin{aligned}
\chi\left(X_{k}\right)=1-\sum_{i_{1}} S^{\prime}\left(\Delta\left(E_{i_{1}}\right), \alpha_{i_{1}}\right)+\sum_{i_{1}<i_{2}} S^{\prime}\left(\Delta\left(E_{i_{1}} E_{i_{2}}\right), \alpha_{i_{1}}+\alpha_{i_{2}}\right)-\cdots \\
\quad+(-1)^{k} S^{\prime}\left(\Delta\left(E_{1} \cdots E_{k}\right), \alpha_{i_{1}}+\cdots+\alpha_{i_{k}}\right) .
\end{aligned}
$$


or equivalently:

$$
\begin{aligned}
\chi\left(X_{k}\right)=1-\sum_{i_{1}} N^{\prime}\left(\tilde{\Delta}\left(E_{i_{1}}\right), \alpha_{i_{1}}\right)+\sum_{i_{1}<i_{2}} & N^{\prime}\left(\tilde{\Delta}\left(E_{i_{1}} E_{i_{2}}\right), \alpha_{i_{1}}+\alpha_{i_{2}}\right)-\cdots \\
& +(-1)^{k} N^{\prime}\left(\tilde{\Delta}\left(E_{1} \cdots E_{k}\right), \alpha_{i_{1}}+\cdots+\alpha_{i_{k}}\right) .
\end{aligned}
$$

Proof. The theorem follows from Theorem 2.20, Corollary 5.6. Theorem 5.11 and Proposition 5.15

Remark 5.30. (1) When the moment polytope map $E \mapsto \Delta(E)$ is additive, that is, $\Delta\left(E_{1} E_{2}\right)=\Delta\left(E_{1}\right)+\Delta\left(E_{2}\right)$ for any two $G$-linear systems $E_{1}, E_{2}$, then the formula (27) can be computed only in terms of the polytopes $\Delta\left(E_{i}\right)$ (see Sections 6.1 and 6.2).

(2) Similarly, when the map $E \mapsto \tilde{\Delta}(E)$ is additive, that is, $\tilde{\Delta}\left(E_{1} E_{2}\right)=\tilde{\Delta}\left(E_{1}\right)+\tilde{\Delta}\left(E_{2}\right)$ for any two $G$-linear systems $E_{1}, E_{2}$, then the formula (28) can be computed only in terms of the polytopes $\tilde{\Delta}\left(E_{i}\right)$ (see Sections 6.1 and 6.2).

The following is the extension of Definition 3.8 to the spherical case:

Definition 5.31. Let $E_{1}, \ldots, E_{k}$ be as above. We say that a nonnegative integer $i$ is critical for the $E_{1}, \ldots, E_{k}$ if there is a nonempty set $J \subset\{1, \ldots, k\}$ such that $N^{\circ}\left(\tilde{\Delta}_{J}\right)>0$ and $\operatorname{dim}\left(\tilde{\Delta}_{J}\right)-|J|=i$ (equivalently $\operatorname{dim}\left(\Delta_{J}\right)+d_{J}-|J|=i$ ). Recall that $\tilde{\Delta}_{J}$ (respectively $\Delta_{J}$ ) is the Newton-Okounkov polytope (respectively the moment polytope) of the linear system $E_{J}=\prod_{i \in J} E_{i}$.

Theorem 5.32 ( $h^{p, 0}$ numbers of a complete intersection in a spherical homogeneous space). Let $p$ be a nonnegative integer which is not critical for $E_{1}, \ldots, E_{k}$ in the sense of Definition 5.31. Then:

$$
h^{p, 0}\left(X_{k}\right)= \begin{cases}1 & p=0 \\ 0 & p \neq 0\end{cases}
$$

Proof. Follows from Theorem 5.3 and Theorem 2.21

Corollary 5.33. Suppose all the numbers $0 \leq i<n-k$ are not critical for $E_{1}, \ldots, E_{k}$. In particular, this is the case if all the Newton-Okounkov polytopes $\tilde{\Delta}_{1}, \ldots, \tilde{\Delta}_{k}$ have full dimension $n=\operatorname{dim}(G / H)$. Then all the numbers $h^{i, 0}\left(X_{k}\right), 0 \leq i \leq n-k$, are zero except for $h^{0,0}\left(X_{k}\right)=1$ and $h^{n-k, 0}\left(X_{k}\right)$ which can be computed from (27) or (28).

Proof. Under the assumptions in the corollary we have $d(J) \geq n-k$ and hence no $p$ with $1 \leq p<n-k$ is a critical number and hence $h^{p, 0}\left(X_{k}\right)=0$ by Theorem 5.32 .

\section{EXAMPLES}

6.1. Horospherical varieties. A subgroup $H \subset G$ is called horospherical if it contains a maximal unipotent subgroup. The corresponding homogeneous space $G / H$ is called horospherical homogeneous space. It can be shown that a horospherical homogeneous space is spherical, that is, it has an open $B$-orbit. A spherical $G$-variety $X$ is horospherical if the open $G$-orbit is horospherical.

It is well-known that:

Proposition 6.1. A subgroup $H \subset G$ is horospherical if and only if there is a parabolic subgroup $P \subset G$ such that $P^{\prime} \subset H \subset P$ (see Kaveh-Khovanskii11, Section 2.1] for a proof). 
Example 6.2. Let $U$ be a maximal unipotent subgroup of $G$. Then $G / U$ is a quasi-affine horospherical homogeneous space. The natural map $G / U \rightarrow G / B$ is a fibration over the flag variety $G / B$ and the fibers are isomorphic to the maximal torus $T$. If $G=\operatorname{GL}(n, \mathbb{C})$, $U$ can be taken to be the subgroup of upper-triangular matrices with 1's on the diagonal.

Remark 6.3. The name horospherical comes from hyperbolic geometry (see Timashev06, Example 7.1]).

Let $\lambda \in \Lambda^{+}$. For a rational $G$-module $M$ let us denote the $\lambda$-isotypic component of $M$ by $M_{\lambda}$, that is, $M_{\lambda}$ is the sum of all the copies of the irreducible $G$-module $V_{\lambda}$ in $M$. Clearly, if $M$ is multiplicity free then $M_{\lambda}$ is either $\{0\}$ or isomorphic to $V_{\lambda}$. In particular, if $\mathcal{E}$ is a $G$-linearized line bundle over a spherical homogeneous space $G / H$ then $H^{0}(G / H, \mathcal{E})_{\lambda}$ is either $\{0\}$ or $V_{\lambda}$. The following is well-known (see Popov86):

Theorem 6.4. Let $G / H$ be a horospherical homogeneous space. Let $\mathcal{E}_{1}$ and $\mathcal{E}_{2}$ be $G$ linearized line bundles on $G / H$ with $E_{i} \subset H^{0}\left(G / H, \mathcal{E}_{i}\right), i=1,2$, two $G$-invariant linear systems. Then for any $\lambda, \gamma \in \Lambda$ the product $\left(E_{1}\right)_{\lambda}\left(E_{2}\right)_{\gamma}$ lies in $\left(E_{1} E_{2}\right)_{\lambda+\gamma}$, where $E_{1} E_{2}$ is the linear system in $\mathcal{E}_{1} \otimes \mathcal{E}_{2}$ spanned by all the products $f_{1} f_{2}, f_{i} \in E_{i}$, and $\left(E_{1} E_{2}\right)_{\lambda+\gamma}$ is the $(\lambda+\gamma)$-isotypic component of $E_{1} E_{2}$.

Let $\mathcal{E}$ be a $G$-linearized line bundle on $G / H$ and take a finite subset $\mathcal{A}$ :

$$
\mathcal{A} \subset \operatorname{Spec}_{G}\left(H^{0}(G / H, \mathcal{E})\right)=\left\{\lambda \mid V_{\lambda} \text { appears in } H^{0}(G / H, \mathcal{E})\right\} .
$$

As before we let $E_{\mathcal{A}}$ denote the linear system:

$$
E_{\mathcal{A}}=\bigoplus_{\lambda \in \mathcal{A}} V_{\lambda}
$$

As in Kaveh-Khovanskii11 one shows that:

Corollary 6.5. With notation as above, the moment polytope of the graded algebra $\overline{\bigoplus_{m} E_{\mathcal{A}}^{m}}$ coincides with the convex hull of $\mathcal{A}$.

Definition 6.6. For each finite subset $\mathcal{A} \subset \Lambda$ let $\Delta(\mathcal{A})$ denote the convex hull of $\mathcal{A}$. In fact $\Delta(\mathcal{A})$ is the moment polytope $\Delta\left(E_{\mathcal{A}}\right)$ of its associated linear system. Also

$$
\tilde{\Delta}(\mathcal{A})=\bigcup_{\lambda \in \Delta(\mathcal{A})}\{\lambda\} \times \Delta_{\underline{w}_{0}}(\lambda),
$$

the corresponding Newton-Okounkov polytope. Recall that $\underline{w}_{0}$ is a fixed reduced decomposition for the longest element $w_{0}$ in the Weyl group $W$ and $\Delta_{\underline{w}_{0}}(\lambda) \subset \mathbb{R}^{N}$ is the string polytope associated to $\lambda$ and $\underline{w}_{0}$ (see Section [5.4).

From Theorem 6.4 it follows that for any two finite subsets $\mathcal{A}_{1}, \mathcal{A}_{2}$ we have $E_{\mathcal{A}_{1}} E_{\mathcal{A}_{2}}=$ $E_{\mathcal{A}_{1}+\mathcal{A}_{2}}$. As in Kaveh-Khovanskii11 we have the following:

Proposition 6.7 (Additivity of the moment and Newton-Okounkov polytopes). (i) The map $E \mapsto \Delta(E)$, which associates to an invariant linear system $E$ its moment polytope, is additive. This is basically the condition (II) in Section 5.5 .

(ii) Suppose the string polytope map $\lambda \mapsto \Delta_{\underline{w}_{0}}(\lambda)$ is additive (e.g. $G=\mathrm{GL}(n, \mathbb{C})$ and Gelfand-Zetlin polytopes). Then the map $E \mapsto \tilde{\Delta}(E)$ is also additive. This is basically the condition (I) in Section 5.5 . 
Let $\mathcal{E}_{1}, \ldots, \mathcal{E}_{k}$ be $G$-linearized line bundles. For each $i=1, \ldots, k$ let $\mathcal{A}_{i}$ be a finite subset of $\operatorname{Spec}_{G}\left(H^{0}\left(G / H, \mathcal{E}_{i}\right)\right) \subset \Lambda^{+}$and $E_{i} \subset H^{0}\left(G / H, \mathcal{E}_{i}\right)$ the corresponding $G$-invariant linear system. Also to simplify the notation, for each $i$ let $\Delta_{i}$ denote the convex polytope $\Delta\left(\mathcal{A}_{i}\right)$, i.e. the convex hull of the finite set $\mathcal{A}_{i}$.

Corollary 6.8 (Genus of a complete intersection in a horospherical homogenous space). Let $f_{1}, \ldots, f_{k}$ be generic elements in $E_{1}, \ldots, E_{k}$ respectively. For each $i=1, \ldots, k$ let $D_{i}=\left\{x \in G / H \mid f_{i}(x)=0\right\}$ be the hypersurface defined by $f_{i}$ and let $X_{k}=D_{1} \cap \cdots \cap D_{k}$. Then:

$$
\begin{aligned}
\chi\left(X_{k}\right)=1-\sum_{i_{1}} S^{\prime}\left(\Delta_{i_{1}}, \alpha_{i_{1}}\right)+\sum_{i_{1}<i_{2}} S^{\prime}\left(\Delta_{i_{1}}+\Delta_{i_{2}}, \alpha_{i_{1}}+\alpha_{i_{2}}\right)-\cdots \\
\quad+(-1)^{k} S^{\prime}\left(\Delta_{1}+\cdots+\Delta_{k}, \alpha_{1}+\cdots+\alpha_{k}\right) .
\end{aligned}
$$

Remark 6.9. As in Remark 5.30(2) if the string polytopes are additive for the choice of the reduced decomposition $\underline{w}_{0}$ (e.g. $G=\operatorname{GL}(n, \mathbb{C})$ and Gelfand-Zetlin polytopes) then the Newton-Okounkov polytope is also additive. Let $\tilde{\Delta}_{i}$ denote the Newton-Okounkov polytope $\tilde{\Delta}\left(\mathcal{A}_{i}\right)$ associated to the finite subset $\mathcal{A}_{i}$. Then, under the assumption of additivity of the string polytopes, we can rewrite the formula (29) for the genus in terms of the NewtonOkounkov polytopes $\tilde{\Delta}_{i}$ and their sums:

$$
\begin{aligned}
\chi\left(X_{k}\right)=1-\sum_{i_{1}} N^{\prime}\left(\tilde{\Delta}_{i_{1}}, \alpha_{i_{1}}\right)+\sum_{i_{1}<i_{2}} N^{\prime}\left(\tilde{\Delta}_{i_{1}}+\tilde{\Delta}_{i_{2}}, \alpha_{i_{1}}+\alpha_{i_{2}}\right)-\cdots \\
\quad+(-1)^{k} N^{\prime}\left(\tilde{\Delta}_{1}+\cdots+\tilde{\Delta}_{k}, \alpha_{1}+\cdots+\alpha_{k}\right) .
\end{aligned}
$$

In particular if $G / H$ is a quasi-affine variety we can consider the finite $G$-invariant subsets of the ring of regular functions $\mathbb{C}[G / H]$, that is, when we take all the line bundles $\mathcal{E}_{i}$ to be the trivial line bundle. In fact, as we now explain, to each face of the Weyl chamber $\Lambda_{\mathbb{R}}^{+}$there corresponds a quasi-affine horospherical homogeneous space and by Proposition 6.1 every horospherical homogeneous space $G / H$ is a quotient of such a quasi-affine horospherical homogeneous space: Let $\sigma$ be a face of the Weyl chamber $\Lambda_{\mathbb{R}}^{+}$. Let $P$ denote the corresponding parabolic subgroup. When $G=\mathrm{GL}(n, \mathbb{C})$, the parabolic subgroups (up to conjugation) are block upper triangular subgroups. Let $P^{\prime}$ denote the commutator subgroup of $P$. One shows that the homogeneous space $G / P^{\prime}$ is quasi-affine and moreover, the algebra of regular functions $\mathbb{C}\left[G / P^{\prime}\right]$, for the natural action of $G$, decomposes as follows ( Popov-Vinberg72]):

$$
\mathbb{C}\left[G / P^{\prime}\right]=\bigoplus_{\lambda \in \sigma \cap \Lambda^{+}} V_{\lambda}^{*}
$$

Now each finite subset $\mathcal{A} \subset \sigma \cap \Lambda^{+}$determines a $G$-invariant subspace $L_{\mathcal{A}}=\bigoplus_{\lambda \in \mathcal{A}} V_{\lambda}$ of $\mathbb{C}\left[G / P^{\prime}\right]$. Let us take finite subsets $\mathcal{A}_{1}, \ldots, \mathcal{A}_{k}$ of the semigroup $\sigma \cap \Lambda$. Then Corollary 6.8 gives us a formula for the genus of a generic complete intersection from the subspaces $L_{1}, \ldots, L_{k}$ in the quasi-affine variety $G / P^{\prime}$, in terms of the convex hulls $\Delta_{i}$ of the subsets $\mathcal{A}_{i}$.

Finally, below is a concrete example of Corollary 6.8.

Example 6.10. Let $V$ be a finite dimensional $G$-module. Let $v_{1}, \ldots, v_{s}$ be highest weight vectors of $V$ with highest weights $\lambda_{1}, \ldots, \lambda_{s}$ respectively. Put $v=v_{1}+\cdots+v_{s}$ and let $X$ be the closure of the $G$-orbit of $v$ in $V$. It is an affine horospherical subvariety of $V$. Let $L$ be the linear subspace of $\mathbb{C}[X]$ consisting of linear functions in $V^{*}$ restricted to $X$. As above one observes that the moment polytope $\Delta$ of the subspace $L$, or equivalently the 
graded algebra $\bigoplus_{m} \overline{L^{m}}$, is $\Delta=\operatorname{conv}\left\{\lambda_{i} \mid i=1, \ldots, s\right\}$. Also let $\tilde{\Delta}$ denote the corresponding Newton-Okounkov polytope. Then if $f$ is a generic element in $L$ defining a hypersurface $H_{f}=\{x \in X \mid f(x)=0\}$, Corollary 6.8 implies that the genus of $H_{f}$ is equal to $S^{\circ}(\Delta)=$ $N^{\circ}(\tilde{\Delta})$, i.e. the number of integral points in the interior of the polytope $\tilde{\Delta}$.

6.2. Group embeddings. Let $\pi: G \rightarrow \mathrm{GL}(n, \mathbb{C}) \subset \operatorname{Mat}(V)$ be a finite dimensional representation of a connected reductive group $G$. Let $\pi_{i j}: G \rightarrow \mathbb{C}, i, j=1, \ldots, n$ be the matrix elements, i.e. the entries of $\pi$. Let $L_{\pi}$ be the subspace of regular functions on $G$ spanned by the $\pi_{i j}$. Consider the action of $G \times G$ on $G$ by the multiplication from left and right. The subspace $L_{\pi}$ is a $(G \times G)$-invariant subspace. Let $\Lambda_{\mathbb{R}}^{+}$(respectively $W$ ) denote the positive Weyl chamber (respectively the Weyl group) of $G$.

Definition 6.11 (Weight polytope). The convex hull of the Weyl orbit of the highest weights of the representation $\pi$ is called the weight polytope of $\pi$. We will denote the weight polytope by $P_{\pi}$ and its intersection with the positive Weyl chamber by $P_{\pi}^{+}$.

As in Kazarnovskii87, one can show that:

Theorem 6.12. The moment polytope of the $(G \times G)$-algebra $\overline{\bigoplus_{m} L_{\pi}^{m}}$ (which lives in $\Lambda_{\mathbb{R}}^{+} \times$ $\left.\Lambda_{\mathbb{R}}^{+}\right)$can be identified with $P_{\pi}^{+}$. To identify the moment polytope and the polytope $P_{\pi}^{+}$we should send a point $\left(\lambda, \lambda^{*}\right)$ to $\lambda$.

Let $F$ denote the Weyl polynomial for the group $G \times G$. It is a polynomial on the vector space $\Lambda_{\mathbb{R}} \times \Lambda_{\mathbb{R}}$ (see Section 5.3. paragraph after Theorem 5.9 for the definition of the Weyl polynomial). For any dominant weight of $G \times G$ of the form $\left(\lambda, \lambda^{*}\right)$ we have $F\left(\lambda, \lambda^{*}\right)=\operatorname{dim}\left(V_{\lambda} \times V_{\lambda}^{*}\right)=\operatorname{dim}\left(V_{\lambda}\right)^{2}$.

Let us take $k$ representation $\pi_{1}, \ldots, \pi_{k}$ where $k \leq \operatorname{dim}(G)$. For each $\pi_{i}$ let $L_{i}$ (respectively $P_{i}^{+}$) be its subspace of matrix elements (respectively its weight polytope intersected with the positive Weyl chamber).

Corollary 6.13 (Genus of a complete intersection in a group). Let $f_{1}, \ldots, f_{k}$ be generic elements in $L_{\pi_{1}}, \ldots, L_{\pi_{k}}$ respectively. For each $i=1, \ldots, k$ let $D_{i}=\{x \in G / H \mid f(x)=0\}$. and let $X_{k}=D_{1} \cap \cdots \cap D_{k}$. Then:

$$
\begin{array}{ll}
\chi\left(X_{k}\right)=1-\sum_{i_{1}} S^{\prime}\left(P_{1}^{+}\right)+\sum_{i_{1}<i_{2}} S^{\prime}\left(P_{1}^{+}+P_{2}^{+}\right)-\cdots & \\
& +(-1)^{k} S^{\prime}\left(P_{1}^{+}+\cdots P_{k}^{+}\right) .
\end{array}
$$

As usual to a representation $\pi$ we can associate a Newton-Okounkov polytope $\tilde{\Delta}_{\pi}$ defined by:

$$
\tilde{\Delta}_{\pi}=\bigcup_{\lambda \in P_{\pi}^{+}}\left(\left\{\left(\lambda, \lambda^{*}\right)\right\} \times \Delta_{\underline{w}_{0}}(\lambda) \times \Delta_{\underline{w}_{0}}\left(\lambda^{*}\right)\right) .
$$

As in Kaveh-Khovanskii10 we have the following:

Proposition 6.14 (Additivity of the moment and Newton-Okounkov polytopes). The map $\pi \mapsto P_{\pi}^{+}$, which associates to a representation $\pi$ its polytope $P_{\pi}^{+}$, is additive with respect to the tensor product of representations. This is basically the condition (II) in Section 5.5 .

(ii) Suppose the string polytope map $\lambda \mapsto \Delta_{\underline{w}_{0}}(\lambda)$ is additive (e.g. $G=\mathrm{GL}(n, \mathbb{C})$ and the Gelfand-Zetlin polytopes). Then the map $\pi \mapsto \tilde{\Delta}_{\pi}$ is also additive. This is basically the condition (I) in Section 5.5. 
As in (ii) above, suppose the string polytope map is additive. Let $\tilde{\Delta}_{i}$ denote the NewtonOkounkov polytope of the representaiton $\pi_{i}$ in Corollary 6.13. Then we can rewrite the formula (31) for the genus in terms of the Newton-Okounkov polytopes $\tilde{\Delta}_{i}$ and their sums:

$$
\begin{aligned}
\left.\chi\left(X_{k}\right)=1-\sum_{i_{1}} N^{\prime}\left(\tilde{\Delta}_{i}\right)+\sum_{i_{1}<i_{2}} N^{\prime}\left(\tilde{\Delta}_{i_{1}}+\tilde{\Delta}_{i_{2}}\right)\right)-\cdots & \\
& +(-1)^{k} N^{\prime}\left(\tilde{\Delta}_{1}+\cdots+\tilde{\Delta}_{k}\right) .
\end{aligned}
$$

6.3. Flag varieties. Let $X=G / B$ be the complete flag variety of a connected complex reductive algebraic group.

Corollary 6.15 (Genus of a complete intersection in the flag variety). Let $\lambda_{1}, \ldots, \lambda_{k}$ be dominant weights. Let $D_{1}, \ldots, D_{k}$ be smooth and transversely intersecting divisors for the corresponding line bundles $L_{\lambda_{1}}, \ldots, L_{\lambda_{k}}$. Let $X_{k}=D_{1} \cap \cdots \cap D_{k}$. Then:

$$
\begin{aligned}
\chi\left(X_{k}\right)=1-\sum_{i_{1}} N^{\prime}\left(\Delta_{\underline{w}_{0}}\left(\lambda_{i_{1}}\right)\right)+\sum_{i_{1}<i_{2}} N^{\prime}\left(\Delta _ { \underline { w } _ { 0 } } \left(\lambda_{i_{1}}\right.\right. & \left.\left.+\lambda_{i_{2}}\right)\right)-\cdots \\
& +(-1)^{k} N^{\prime}\left(\Delta_{\underline{w}_{0}}\left(\lambda_{1}+\cdots+\lambda_{k}\right)\right) .
\end{aligned}
$$

Finally we briefly discuss the case of the flag variety of $G=\mathrm{GL}(n, \mathbb{C})$ and Gelfand-Zetlin polytopes. Let $G=\mathrm{GL}(n, \mathbb{C})$. The flag variety of $G$ can be identified with the variety of all flags of linear subspace in $\mathbb{C}^{n}$ :

$$
\{0\} \varsubsetneqq F_{1} \varsubsetneqq \cdots \varsubsetneqq F_{n}=\mathbb{C}^{n} .
$$

Each dominant weight $\lambda$ of $G$ can be represented as an increasing $n$-tuple of integers:

$$
\lambda=\left(\lambda_{1} \leq \cdots \leq \lambda_{n}\right) .
$$

In their well-known work Gelfand-Zetlin50, given a dominant weight $\lambda$, Gelfand and Zetlin construct a natural vector basis for the irreducible representation $V_{\lambda}$ whose elements are parameterized with the integral points $x_{i, j}$ satisfying the following set of interlacing inequalities:

$$
\begin{aligned}
& \begin{array}{llllll}
\lambda_{1} & \lambda_{2} & \ldots & \ldots & \ldots & \lambda_{n}
\end{array} \\
& \begin{array}{lllll}
x_{1, n-1} & x_{2, n-1} & \cdots & \cdots & x_{n-1, n-1}
\end{array} \\
& x_{2, n-2} \quad x_{2, n-2} \quad \cdots \quad x_{n-2, n-2} \\
& x_{1,2} \quad x_{2,2} \\
& x_{1,1}
\end{aligned}
$$

where the notation

$$
a \quad b
$$

means $a \leq c \leq b$.

The set of all points $\left(x_{i, j}\right)$ in $\mathbb{R}^{n(n-1) / 2}$ satisfying (34) is called the Gelfand-Zetlin polytope associated to $\lambda$ denoted by $\Delta_{\mathrm{GZ}}(\lambda)$. Let $\mathcal{L}_{\lambda}$ be the $G$-line bundle on the flag variety associated to a dominant weight $\lambda$. If $H$ is a generic divisor of $\mathcal{L}_{\lambda}$ then Corollary 6.15 states that the genus of $H$ is equal to the number of integral points in $\mathbb{R}^{n(n-1) / 2}$ lying in 
the interior of $\Delta_{\mathrm{GZ}}(\lambda)$. In other words, the number of integral points in $\mathbb{R}^{n(n-1) / 2}$ which satisfy the inequalities in (34) where all the inequalities are strict.

\section{REFERENCES}

[Alexeev-Brion04] Alexeev, V.; Brion, M. Toric degeneration of spherical varities. Selecta Math. (N.S.) 10 (2004), no. 4, 453-478.

[Bernstein75] Bernstein, D. N. The number of roots of a system of equations. English translation: Functional Anal. Appl. 9 (1975), no. 3, 183-185 (1976).

[Berenstein-Zelevinsky01] Berenstein, A.; Zelevinsky, A. Tensor product multiplicities, canonical bases and totally positive varieties. Invent. Math. 143 (2001), no. 1, 77-128.

[Brion89] Brion, M. Groupe de Picard et nombres caracteristiques des varieties spheriques. Duke Math. J. 58 (1989), no. 2, 397-424.

[Brion87] Brion, M. Sur l'image de l'application moment. Séminaire d'algèbre Paul Dubreil et Marie-Paule Malliavin (Paris, 1986), 177-192, Lecture Notes in Math., 1296, Springer, Berlin, 1987.

[Brion90] Brion, M. Une extension du théorème de Borel-Weil. Math. Ann. 286 (1990), no. 4, 655-660.

[Carrell-Liebermann73] Carrell, J. B.; Lieberman, D. I. Holomorphic vector fields and Kaehler manifolds, Invent. Math. 21 (1973), 303-309.

[DeConcini-Procesi82] De Concini, C., and C. Procesi, Complete symmetric varieties. Invariant theory (Montecatini, 1982), Lecture Notes in Math., 996, Springer, Berlin (1983), 1-44.

[Gelfand-Zetlin50] Gelfand, I.M.; Cetlin, M.L. Finite dimensional representations of the group of unimodular matrices, Doklady Akad. Nauk USSR (N.S.) ,71 (1950), 825-828.

[Guillemin-Sternberg84] Guillemin, V.; Sternberg, S. Geometric quantization and multiplicities of group representations. Invent. Math. 77 (1984), 533-546.

[Hartshorne77] Hartshorne, R. Algebraic geometry. Graduate Texts in Mathematics, No. 52. SpringerVerlag, New York-Heidelberg, 1977.

[Kaveh04] Kaveh, K. Morse theory and Euler characteristic of sections of spherical varieties. Transform. Groups 9 (2004), no. 1, 47-63.

[Kaveh] Kaveh, K. Crystal bases and Newton-Okounkov bodies. To appear in Duke Mathematical Journal.

[Kaveh-Khovanskii10] Kaveh, K.; Khovanskii, A. G.Moment polytopes, semigroup of representations and Kazarnovskii's theorem. J. Fixed Point Theory Appl. 7 (2010), no. 2, 401-417.

[Kaveh-Khovanskii11] Kaveh, K.; Khovanskii, A. G. Newton polytopes for horospherical spaces. Mosc. Math. J. 11 (2011), no. 2, 265-283, 407.

[Kaveh-Khovanskii12a] Kaveh, K.; Khovanskii, A. G. Newton-Okounkov bodies, semigroups of integral points, graded algebras and intersection theory. Ann. of Math. (2) 176 (2012), no. 2, 925-978.

[Kaveh-Khovanskii12b] Kaveh, K.; Khovanskii, A. G. Convex bodies associated to actions of reductive groups. Moscow Mathematical Journal, 12 (2012) no. 2., 369-396, 461.

[Kazarnovskii87] Kazarnovskii, B. Newton polyhedra and the Bezout formula for matrix-valued functions of finite dimensional representations. Functional Analysis and its applications, v. 21, no. 4, 73-74 (1987).

[Khovanskii77] Khovanskii, A. G., Newton polyhedra and toroidal varieties. Funkcional. Anal. i Priložen. 11 (1977), no. 4, 56-64, 96.

[Khovanskii78] Khovanskii, A. G., Newton polyhedra and the genus of complete intersections. Funktsional. Anal. i Prilozhen. 12 (1978), no. 1, 51-61.

[Khovanskii15] Khovanskii, A. G. Newton polyhedra and irreducible components of complete intersections. To appear in Izvestiya RAN, Ser. Matematika (2015).

[Khovanskii-Pukhlikov93] Khovanskii, A. G.; Pukhlikov, A. V. Finitely additive measures of virtual polyhedra. (Russian) Algebra i Analiz 4 (1992), no. 2, 161-185; translation in St. Petersburg Math. J. 4 (1993), no. 2, 337-356

[Kiritchenko06] Kiritchenko, V. Chern classes of reductive groups and an adjunction formula. Ann. Inst. Fourier (Grenoble) 56 (2006), no. 4, 1225-1256.

[Kiritchenko07] Kiritchenko, V. On intersection indices of subvarieties in reductive groups. Mosc. Math. J. 7 (2007), no. 3, 489-505, 575.

[Kushnirenko76] Kushnirenko, A. G. Polyedres de Newton et nombres de Milnor. (French) Invent. Math. 32 (1976), no. 1, 1-31.

[Lazarsfeld-Mustata09] Lazarsfeld, R.; Mustata, M. Convex bodies associated to linear series. Annales scientifiques de l'ENS 42, no. 5 (2009), 783-835.

[Littelmann98] Littelmann, P. Cones, crystals, and patterns. Transform. Groups 3 (1998), no. 2, 145-179. 
[McMullen77] McMullen, P. Valuations and Euler-type relations on certain classes of convex polytopes. Proc. London Math. Soc. (3) 35 (1977), no. 1, 113-135.

[Ness84] Ness, L. A stratification of the null cone via the moment map. With an appendix by David Mumford. Amer. J. Math. 106 (1984), no. 6, 1281-1329.

[Okounkov96] Okounkov, A. Brunn-Minkowski inequality for multiplicities. Invent. Math. 125 (1996), no. $3,405-411$.

[Okounkov97] Okounkov, A. A remark on the Hilbert polynomial of a spherical variety. Func. Anal. and Appl., 31 (1997), 82-85.

[Okounkov03] Okounkov, A. Why would multiplicities be log-concave? The orbit method in geometry and physics (Marseille, 2000), 329-347, Progr. Math., 213, Birkha"user Boston, Boston, MA, 2003.

[Perrin14] Perrin, N. On the geometry of spherical varieties. Transform. Groups 19 (2014), no. 1, 171-223.

[Popov86] Popov, V. L. Contractions of actions of reductive algebraic groups. Mat. Sb. (N.S.) 130(172) (1986), no. 3, 310-334, 431

[Popov-Vinberg72] Popov, V. L.; Vinberg, E. B. A certain class of quasihomogeneous affine varieties. (Russian) Izv. Akad. Nauk SSSR Ser. Mat. 36 (1972), 749-764.

[Samuel-Zariski60] Samuel, P.; Zariski, O. Commutative algebra. Vol. II. Reprint of the 1960 edition. Graduate Texts in Mathematics, Vol. 29.

[Timashev06] Timashev, D. Homogeneous spaces and equivariant embeddings.

[Viro88] Viro, O. Ya. Some integral calculus based on Euler characteristic. Topology and geometry-Rohlin Seminar, 127-138, Lecture Notes in Math., 1346, Springer, Berlin, 1988.

Department of Mathematics, University of Pittsburgh, Pittsburgh, PA, USA.

E-mail address: kaveh@pitt.edu

Department of Mathematics, University of Toronto, Toronto, Canada; Moscow Independent University, Moscow, Russia.

E-mail address: askold@math.utoronto.ca 